\title{
Knowledge-Based Shape Optimization of Morphing Wing for More Efficient Aircraft
}

\author{
Alessandro De Gaspari and Sergio Ricci \\ Department of Aerospace Science and Technology, Politecnico di Milano, Via La Masa 34, 20156 Milano, Italy \\ Correspondence should be addressed to Sergio Ricci; sergio.ricci@polimi.it
}

Received 31 March 2015; Revised 22 July 2015; Accepted 31 August 2015

Academic Editor: Ning Qin

Copyright ( 2015 A. De Gaspari and S. Ricci. This is an open access article distributed under the Creative Commons Attribution License, which permits unrestricted use, distribution, and reproduction in any medium, provided the original work is properly cited.

\begin{abstract}
An optimization procedure for the shape design of morphing aircraft is presented. The process is coupled with a knowledgebased framework combining parametric geometry representation, multidisciplinary modelling, and genetic algorithm. The parameterization method exploits the implicit properties of the Bernstein polynomial least squares fitting to allow both local and global shape control. The framework is able to introduce morphing shape changes in a feasible way, taking into account the presence of structural parts, such as the wing-box, the physical behaviour of the morphing skins, and the effects that these modifications have on the aerodynamic performances. It inherits CAD capabilities of generating 3D deformed morphing shapes and it is able to automatically produce aerodynamic and structural models linked to the same parametric geometry. Dedicated crossover and mutation strategies are used to allow the parametric framework to be efficiently incorporated into the genetic algorithm. This procedure is applied to the shape design of Reference Aircraft (RA) and to the assessment of the potential benefits that morphing devices can bring in terms of aircraft performances. It is adopted for the design of a variable camber morphing wing to investigate the effect of conformal leading and trailing edge control surfaces. Results concerning four different morphing configurations are reported.
\end{abstract}

\section{Introduction}

The very challenging targets of new environmental requirements for transport aircraft force the researchers to look for more advanced aircraft configurations, based on more efficient aerodynamics and structures together with more sophisticated flight control systems. Focusing on European transport, it appears as clear that the pressure will increase for large scheduled European carriers to reequip their short haul fleets with more fuel-efficient types, in order to remain competitive with low-cost rivals and to ensure they will not be unduly penalized when European emissions trading comes into full force.

The Bréguet range equation $[1,2]$ combines aerodynamic, propulsion, and structural figures of merit and suggests acting on both the aircraft empty weight and the lift over drag ratio, in order to improve the modern aircraft efficiency. Many are the approaches investigated during recent years trying to improve all the Bréguet equation terms, such as advanced and unconventional configurations able to improve aircraft efficiency, new materials and structural concepts, active controls to peak loads reduction and flight control improvements, more efficient engines, and alternative fuels [3]. Certainly morphing technologies offer potential benefits for a more efficient aircraft and for this reason literature reports many and different morphing concepts even if a clear evaluation of their real benefits is not available yet [47]. One of the key challenges for morphing technologies is represented by the identification of the most suitable actuation concept able to reduce the actuation energy and the mechanism weight.

While current aircraft are already equipped with systems able to introduce in-flight geometrical variations such as wing area change, variable camber, and retractable landing gear, the morphing of next generation still has challenges and leads to the design of morphing wings based on conformable control surfaces [8]. In particular, the variable wing camber morphing, considered as the capability to change the airfoil 
shape without surface discontinuities often based on the adoption of ad hoc designed flexible skins [9], appears as an efficient way to maximize the lift over drag ratio and to reduce the fuel consumption over the entire flight envelope. However, the design of this kind of morphing devices requires developing specific procedures able to assist the engineers during both the design [10-12] and the benefits evaluation phases. Currently, this target is commonly addressed by means of dedicated multilevel and multiobjective optimization procedures [13]. Multilevel capabilities allow performing the optimization of morphing shapes and the design of the morphing mechanism separately [14]; multiobjective techniques help to design aircraft able to adapt its shape to optimize the performances along the cruise or at a wide range of different flight conditions.

The design of morphing wing devices must combine two opposite requirements, often named kinematic and structural requirements, respectively: a flexible structure so to minimize the energy necessary to adapt its shape as expected and at the same time an enough rigid structure able to maintain the new shape under the aerodynamic loads when the morphing mechanism is not actuated. The approach proposed by the authors [15] is based on the optimization of aerodynamic, stiffness, and actuation sequentially, passing through the definition of a family of optimal morphing shapes associated with a group of as many flight conditions. Hence, the design of morphing mechanism depends on the availability of the optimal shapes that must be achieved when it is actuated and that are computed before the mechanism is known. In this way the optimal shapes guarantee the aerodynamic performances, while the mechanism can be optimized considering both kinematic and structural requirements. The key problem is that the allowable shape variation laws depend on the type of mechanism, while the mechanism design depends on the shape changes that the mechanism must be able to reach. For this reason, in recent years many efforts have been made aiming at considering energy and actuation requirements [16-18], as well as structural constraints [19], directly during the aerodynamic shape optimization.

The work presented in this paper focuses on the first level of a wider morphing design framework having the following capabilities: wing shape optimization able to combine aerodynamic performances with optimal deformation of the skins (first level); optimal design of compliant mechanism able to produce, once actuated, the optimal shape coming out from the first level (second level); integration of the morphing devices into a high-fidelity model representing the complete aircraft for final aeroelastic assessment to evaluate the reliability of the designed morphing solutions [20].

Initially, the first level was implemented as a simple $2 \mathrm{D}$ shape optimization linked to a viscous and subsonic $2 \mathrm{D}$ aerodynamic solver, while the second one represented a general code for the synthesis of compliant mechanisms [15, 21] and two software pieces have been developed separately. Recently, the first one has been extended in order to produce $3 \mathrm{D}$ wing models starting from the results which continued to be obtained by $2 \mathrm{D}$ shape optimization while the second one has been improved by adding multiobjective capabilities [22].
The paper describes the novel progress beyond this point about the first level optimization which is now based on a comprehensive knowledge-based engineering (KBE) framework able to define the optimal morphing wing shape in terms of mission profile performances, directly in the three-dimensional space. It is based on coupling a parametric geometry representation, able to predict the structural response of morphing skin, with Computer-Aided Engineering (CAE) capabilities, Object-Oriented Programming (OOP), genetic algorithm, and aerodynamic analyses. The results obtained applying it to a Reference Aircraft (RA), coming from the FP7 EU NOVEMOR project (Novel Air Vehicle Configurations: from Fluttering Wings to Morphing Flight) and adopted as a benchmark to evaluate the optimal benefits that can bring in terms of global performances, are reported to validate the proposed procedure and to evaluate the impact of continuous chordwise and spanwise camber variation in terms of lift to drag ratio and aerodynamic load distribution.

\section{Shape Design Methodology}

The shape design procedure presented in this work consists of a shape optimization, based on genetic algorithm and coupled with the parametric framework introduced in the following section, able to design the optimal morphing shapes from the aerodynamic point of view, under skin structural requirements. While genetic algorithms have been already coupled with 2D shape optimization of airfoil $[23,24]$, this paper presents an approach, based on a particular use of the genetic algorithms, able to combine the set of the most important cross sections describing the wing, in order to define the optimal shape of a 3D morphing wing.

The set of parameterized cross section shapes is the starting point of the whole process shown in Figure 1. The outer optimization loop, based on genetic algorithm, works on the most important design variables affecting the camber morphing, such as the leading and trailing edge deflections. After the cross sections are combined by PHORMA, the code described in Section 3.1, the 3D model is analytically defined and its shape can be easily controlled and used to estimate the aerodynamic performances by means of the user-defined fitness evaluation function. Different performance indices can be enclosed in the shape optimization process in order to improve the aerodynamic efficiency and reduce the fuel consumption or to guarantee that the morphing aircraft has optimal aerodynamic characteristics over different flight conditions $[25,26]$. The inner loop, based on a dedicated development of CST method [27], guarantees that the outer loop works only on feasible shapes able to satisfy wing-box volume constraints and morphing skin structural requirements.

This parametric shape representation is suitable to be incorporated into genetic algorithm and to be parallelized $[28,29]$. The most innovative aspect of this implementation is that the skin structural behaviour, recovered from the adopted geometry representation, determines the rules which drive the reproduction process of the population. In this way, the resulting morphing shape comes from a natural selection 


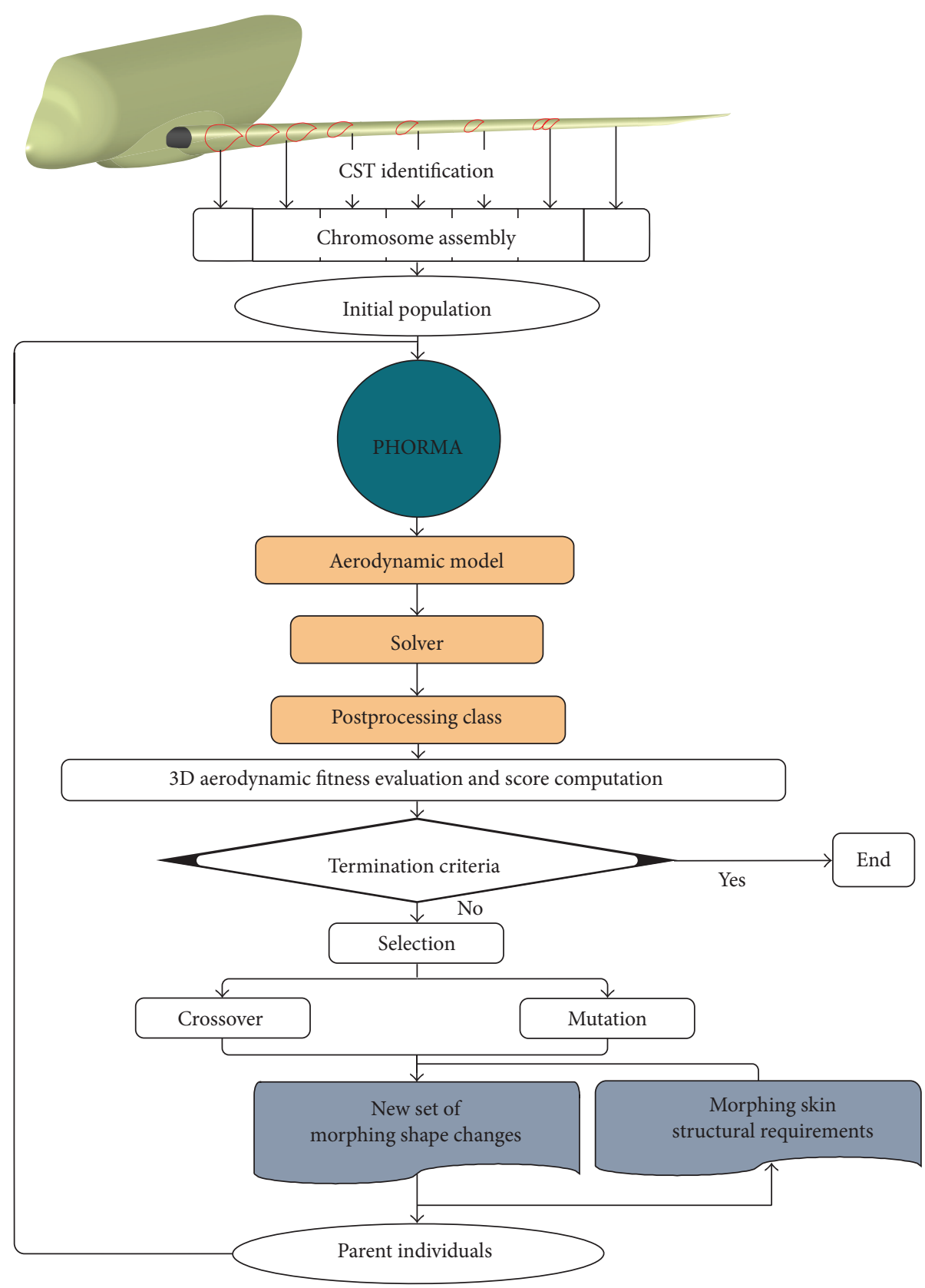

FIGURE 1: Scheme of the genetic algorithm based on the 3D parametric shape representation.

able to take into account the physics related to structural deformation of the skin.

Each individual making up the population is divided in as many parts as the wing sections are and each part is composed of the same set of optimization variables. The generation of each new population is produced by in-house crossover and mutation dedicated strategies, while a code parallelization allows decreasing the computational cost. Two possible code parallelization modes can be adopted. The first one computes in parallel the fitness functions in terms of global aerodynamic performance. The second one divides the wing problem into a number of subproblems, equal to the number of wing sections, that are solved simultaneously.

\section{The Knowledge-Based Framework}

The shape design of morphing aircraft, able to smoothly change their external geometry, requires interfacing the aerodynamics that can be optimized for different flight conditions of the mission profile and the structure of the skin that constitutes the outermost component of the aircraft. CAE systems represent parametric tools, including Finite Element Analysis (FEA) and Computational Fluid Dynamics (CFD), able to assist the engineering in the shape design, but the ability to introduce complex changes is limited and the only rules that can be embedded are restricted to simple geometrical statements. KBE systems are designed to allow implicit 


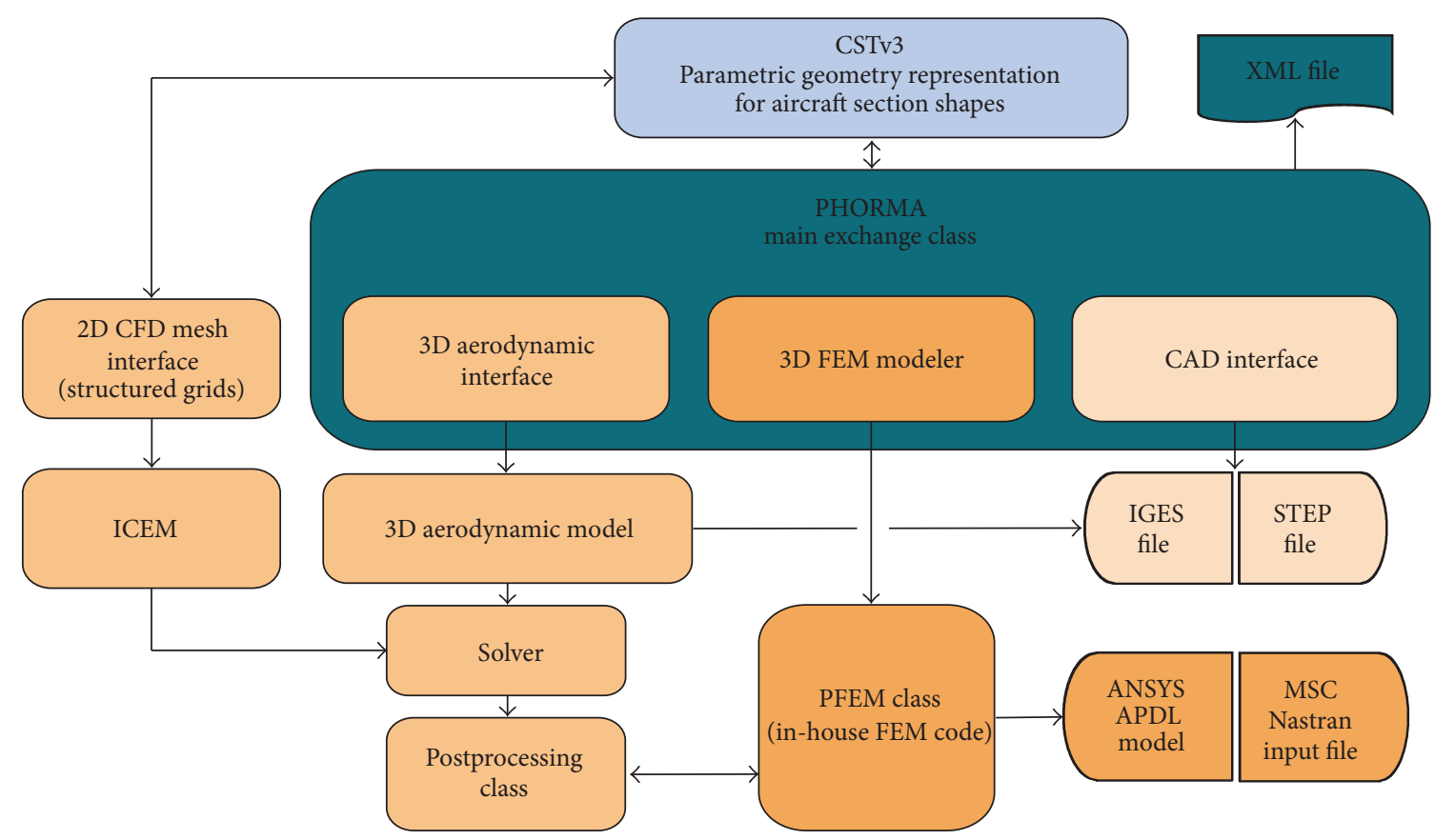

FIgURE 2: The parametric framework.

rules, physics-based solvers, and evolutionary algorithms to be linked to CAD system. A specific knowledge-based framework, able to provide advanced implicit parametric capabilities and more direct shape control than common CAE systems, is here presented. It is a fully integrated multiphysics environment based on a parametric geometry representation of the aircraft that incorporates the ability to predict the structural response of the skin to the shape changes, provides couplings with aerodynamic solvers, and uses genetic algorithm to tackle the optimization problem related to the definition of the best morphing shapes.

The knowledge of the morphing skin structural behaviour is included into a so-called skin structural (KBSS) feedback that consists of an inference engine that uses rules to deduce new shapes consistent with the structural response of the morphing skin. This knowledge-based technology is strictly related to an Object-Oriented Programming-based geometry parameterization, that is, the Class Shape Transformation (CST), which is integrated with a Computer-Aided Engineering (CAE) tool. The integration with $\mathrm{CAD}$ allows enriching the parametric capabilities of the CST formulation with the parametric features of the CAD systems, for a complete and accurate $3 \mathrm{D}$ shape definition. When this knowledge-based system is coupled with genetic algorithm, as described in Section 2, it offers very efficient criteria able to drive the evolution of the solution during the whole optimization process. In this way it acts not only on the results, but also on how the evolutionary process works to reach the final solution.

The architecture of the framework is shown in Figure 2 where the implementation sequence of the three main parts and their mutual interactions are represented. The generation of the $3 \mathrm{D}$ models corresponding to different morphing configurations is done in two steps: the parametric identification of the aircraft and its shape modification according to the KBE strategy. Once a 3D geometry is identified in a parametric way, different FEM, CFD, and CAD models can be linked to a common shape representation. In this way the shape changes can be quickly extended to the physical models.

The structural, aerodynamic, and CAD modelling capabilities are implicitly connected and inherit their properties from a main class able to combine in three dimensions the set of the most important parameterized cross sections of the aircraft model. A specific code directly connected to the CST method is also available for the automatic and high-fidelity $2 \mathrm{D}$ aerodynamic analyses of each cross section.

3.1. Aircraft Shape Parameterization. The KBE framework revolves around PHORMA (Parametric sHapes for aerOdynamic and stRuctural Modelling of Aircraft) that is an object oriented code composed by a suite of modules conceived to exchange and handle different shapes, corresponding to the set of the most important cross sections of the aircraft model, in order to generate the 3D geometry. The shapes are parameterized and combined in the $3 \mathrm{D}$ space through CAD-based interpolation techniques so that local shapes changes can be spread out. Resulting geometry is shared by the aerodynamic, structural, and CAD models that inherit the parametric capabilities from the so-called class/shape function transformation (CST) technique, originally proposed by Kulfan [27] and extended by PoliMi to morphing wing sections [21]. The CST parameterization is based on merging four terms: a shape function, a class function, and two additional terms related to the airfoil leading edge and trailing edge shapes. 


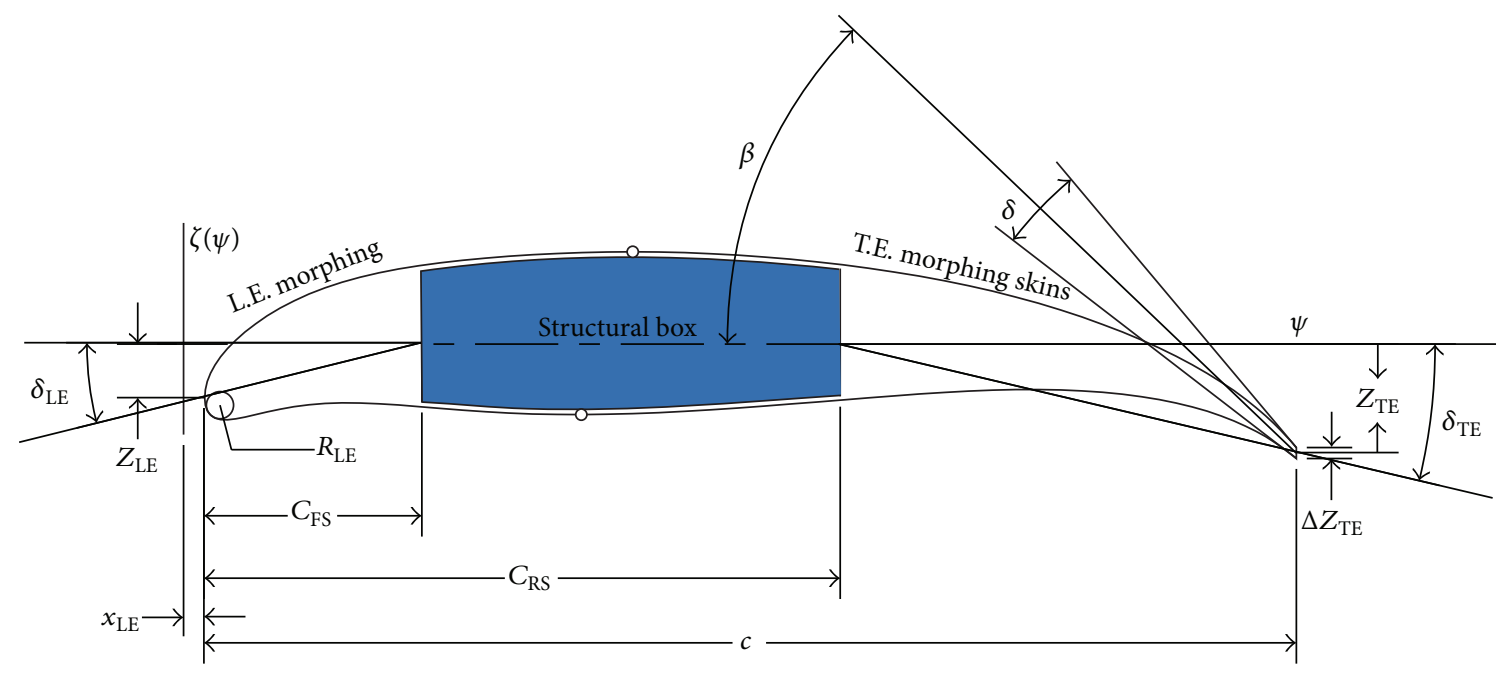

FIGURE 3: CSTv3 geometric parameters for airfoil sections.

The general mathematical expression representing the airfoil geometry, already presented in [15], is here defined as

$$
\zeta(\psi)=C_{N 2}^{N 1}(\psi) \mathbf{a} \cdot \mathbf{b}_{n}^{T}(\psi)+\psi \zeta_{\mathrm{TE}}+(1-\psi) \zeta_{\mathrm{LE}}
$$

where $\psi=x / c, \zeta=z / c$ are the nondimensional coordinates with respect to airfoil chord $c, \zeta_{\mathrm{TE}}=Z_{\mathrm{TE}} / c$, and $\zeta_{\mathrm{LE}}=$ $Z_{\mathrm{LE}} / c$. These parameters in addition to the airfoil leading edge nose radius $R_{\mathrm{LE}}$, the trailing edge boat-tail angle $\beta$, the thickness $\Delta Z_{\mathrm{TE}}$, and the angular thickness $\delta$ represent the set of "CST parameters" and are shown in Figure 3. Starting from these quantities, the leading edge deflection is conventionally defined as $\delta_{\mathrm{LE}}=\arctan \left(Z_{\mathrm{LE}} /\left(C_{\mathrm{FS}}-x_{\mathrm{LE}}\right)\right)$, while the trailing edge equivalent deflection can be defined as $\delta_{\mathrm{TE}}=\arctan \left(Z_{\mathrm{TE}} /\left(c-C_{\mathrm{RS}}\right)\right)$, where $C_{\mathrm{FS}}$ and $C_{\mathrm{RS}}$ are the front and rear spar position, respectively. It is noticed that it is different and generally smaller than the boat-tail angle $\beta$.

The first term of (1) is the class function and provides control of both the leading and the trailing edge shape. It includes the exponents $N 1$ and $N 2$ that are used to mathematically define a variety of basic general shapes [15]. The second term of (1) is the shape function that is defined using a set of $(n+1)$ Bernstein polynomial components $B_{i}(\psi)$ (of selected order $n$ ) included in the function array $\mathbf{b}_{n}(\psi)$, scaled by as many unknown extra coefficients $A_{i}$ included in the vector $\mathbf{a}$.

The $i$ th shape function component is defined as the product between the $i$ th Bernstein polynomial component and the $i$ th associated binomial coefficient. Each Bernstein polynomial component is multiplied by the class function defining the systematic decomposition of the airfoil shapes into corresponding scalable airfoil components. Consequently, the constant coefficient $A_{i}$ can be used to scale the entire airfoil function which is a smooth function that eliminates local discontinuities.

The first and last terms of the vector a are related to the leading and trailing edge boundary conditions and are equal to the shape function evaluated in $x / c=0$ and $x / c=1$.
In particular $\mathbf{a}(1)=\sqrt{2 R_{\mathrm{LE}} / c}$ and $\mathbf{a}(n+1)=\tan \beta+Z_{\mathrm{TE}} / c-$ $Z_{\mathrm{LE}} / c$.

The perturbation of one of the extra coefficients proportionally changes the value that the CST function assumes at the chordwise station of the peaks of every airfoil component. They are equally spaced along the airfoil chord and are defined as

$$
\psi_{\max (Z)}=\frac{N 1+i}{N 1+N 2+n} \quad \text { for } i=0, \ldots, n .
$$

Many other properties of the cross-section shapes, such as the airfoil area, the nondimensional arc length nal of the leading edge or the upper and lower surfaces, the airfoil camber, or thickness, can be computed in a parametric way. Thanks to its nature, the calculation of the first- and secondorder derivatives is very fast and can be used to compute geometrical quantities, such as the length $L(x)$ and curvature $\kappa(x)$ of the upper and lower sides, as well as leading edge, surfaces, or a portion of them.

PHORMA is implemented by the Object-Oriented Programming so that different cross-sectional shapes of a complete aircraft can be identified in order to reproduce the corresponding reference shape and to have a 3D mathematical model, based on CST parameterization, suitable to introduce morphing shape changes. In this way the estimation of the morphing skin structural behaviour, as well as the generation of the aerodynamic or finite element models, of the aircraft inherits all methods coming from its parametric representation.

3.2. Parametric Identification. In order to have a parametric model suitable to introduce morphing shape modifications, an efficient CST identification of preexisting CAD models is available. By means of a completely automatic procedure, the shapes corresponding to the set of the most important cross sections of the aircraft model are parameterized and corresponding CST models are generated. The upper and lower extra coefficients ( $A_{\text {up }}$ and $A_{\text {low }}$ ) of each shape function 
can be evaluated by a variety of techniques depending on the particular study, such as numerical optimizations. In this paper, a very efficient CST identification, based on closedform least squares fitting able to match preexisting CAD models, is presented. The procedure is completely automatic and allows catching the arbitrary set of cross sections of the CAD model. Equation (1) can be rearranged as follows:

$$
\left[C_{N 2}^{N 1}(\psi) \mathbf{b}_{n}(\psi)\right] \cdot \mathbf{a}^{T}=\zeta(\psi)-\psi \zeta_{\mathrm{TE}}-(1-\psi) \zeta_{\mathrm{LE}}
$$

The parameterization process is based on a specific least squares fitting, where the Bernstein basis, described by [30], is replaced by the here defined CST basis:

$$
\begin{aligned}
& T_{n} \\
& \quad=\left\{t_{i}^{(n)}=C_{N 2}^{N 1}(\psi)\left(\begin{array}{c}
n \\
i
\end{array}\right)(1-\psi)^{n-i} \psi^{i}, i=0, \ldots, n\right\} .
\end{aligned}
$$

The CST basis is the basis of the space of the class-shape functions of degree less than or equal to $n$ on the interval $[0,1]$. Equation (3) can be expressed in the CST basis as follows:

$$
\mathbf{t}_{n}(\psi) \cdot \mathbf{a}^{T}=f(\psi)
$$

where $\mathbf{t}_{n}(\psi)$ is the vector containing the CST components in the CST basis and $f(\psi)$ is equal to the right terms of (3).

If $\left\{\chi_{j}\right\}_{1 \leq j \leq l+1} \in(0,1)$ are $l+1$ pairwise distinct real points and $\left\{f_{j}\right\}_{1 \leq j \leq l+1} \in \mathbb{R}$, computing the $(n+1)$ coefficients a is equivalent to solve, in the least squares sense, the overdetermined system:

$$
\mathbf{T}_{n} \mathbf{a}^{T}=\mathbf{f}
$$

where $\mathbf{T}_{n}$ is the here defined $(l+1) \times(n+1)$ CST-Vandermonde matrix for the CST basis $T_{n}$ and $\mathbf{f}$ is the $(l+1)$ vector, both evaluated in the nodes $\left\{\chi_{j}\right\}_{1 \leq j \leq l+1}$.

Taking into account that $\mathbf{T}_{n}$ has full rank $(n+1)$, the problem has a unique solution given by the unique solution of the linear system:

$$
\mathbf{T}_{n}^{T} \mathbf{T}_{n} \mathbf{a}^{T}=\mathbf{T}_{n}^{T} \mathbf{f}
$$

Since $\mathbf{T}_{n}$ is usually an ill-conditioned matrix, the $Q R$ factorization can be used to solve the system.

The approximation can be improved extracting the boundary condition terms from the definition of the CST basis and embedding them within the right term of (5). The CST basis is redefined for $i=1, \ldots, n-1$, while $f(\psi)$ is recomputed as

$$
\begin{aligned}
f(\psi)= & \zeta(\psi)-\psi \zeta_{\mathrm{TE}}-(1-\psi) \zeta_{\mathrm{LE}} \\
& -C_{N 2}^{N 1}(\psi)\left[A_{0} B_{0}(\psi)+A_{n} B_{n}(\psi)\right] .
\end{aligned}
$$

The terms $A_{0}$ and $A_{n}$ of (8) are defined by the extra coefficients $\mathbf{a}(1)$ and $\mathbf{a}(n+1)$ and depend on the CST parameters $R_{\mathrm{LE}}, c, \beta, Z_{\mathrm{TE}}$, and $Z_{\mathrm{LE}}$ that can be directly computed starting from the data points.
3.3. Structural Response of Morphing Skin. The parametric framework introduced in this section is a dedicated knowledge-based engineering (KBE) environment able to force the shape design to move inside a domain where the global shape is driven to change in a feasible way. First of all, a morphing wing shape is feasible if it is able to take into account the structural behaviour of the skins. It depends on how the actuation is introduced and how the morphing skin responds to the actuation input together with the external loads. A Knowledge-Based Skin Structural (KBSS) feedback embedded into the shape parameterization scheme allows taking into account the skin structural response before the morphing mechanism has been defined.

One of the most important obstacles in the wing morphing is due to two main aspects:

(i) The presence of main bearing structures.

(ii) The structural contribution of the skin.

Indeed, even if almost all the proposed approaches for morphing wings are based on a different structural configuration of the ribs, the structural contribution of the skin still remains. Moreover the skin is directly connected to the structural box which represents an obstacle for a full deformation and restricts outside deformable regions. In the approach here presented the knowledge-based parameterization is able to take into account all of these aspects in an implicit way. It represents a tool that assists the engineers in determining the best morphing shape to limit the deformation energy of the skin and, at the same time, the actuator power necessary to control the shape change. This approach is general and can be easily applied to skin made of different type of material.

When local perturbations are introduced in a wing shape, one of the main feasible constraints is represented by the wing regions that must be kept undeformed. Considering a conventional wing equipped with active camber, this region corresponds to the structural box. Introducing this equality constraint in an explicit way, for example, during an optimization process, is an expensive approach in terms of computational effort. A different approach is to exploit the CST basis capabilities, described in Section 4.1, not only to handle volume constraints, but also to ensure that the skin fits the fixed parts of the wing. In this way the knowledge-based approach is able to follow a set of implicit rules governing the skin structural behaviour in order to prevent the case in which the shape changes violate the wing-box constraint during the optimization process.

The CST formulation offers the possibility to capture a second structural feedback related to the behavior of the portion of morphing skin outside the undeformed regions. The estimation of the skin structural deformation here proposed is directly inherited from the analytical CST formulation and it is included in the KBSS feedback.

The structural behavior of the skin has a key role in the shape changes that the skin can assume during the deformation. It can be obstacle or a help to achieve the desired shape. This link is preserved by the CST formulation which embeds, in the same parameterization technique, the ability to introduce shape changes and, at the same time, to evaluate 
the corresponding stress distribution along the skin. The first ability has been previously described, while the second one is based on geometrical consideration, already described in [15]. The axial strain $\varepsilon_{\text {axial }}$ (or axial stress $\sigma_{\text {axial }}$ ) required to stretch or compress the skin and the bending deformation $\varepsilon_{\text {bend }}$ (or bending stress $\sigma_{\text {bend }}$ ) can be directly deduced, respectively, by the length $\Delta L(x)$ and curvature $\Delta \kappa(x)$ difference function between the initial and the deformed shape, with respect to the same normalized arc length function $\operatorname{nal}(x)$. If the skin structural properties, such as Young's Modulus $E$ and the skin thickness $t$, are known, the comparison between the CAD models representing the initial and the deformed shapes allows estimating the strain distribution along the upper and lower as well as leading edge skin surfaces.

3.4. Multidisciplinary Modelling. Once a parametric model is available and morphing shape changes are introduced, corresponding CAD, FEM, or CFD models can be linked to the same shape geometry. While the framework described in this section allows exporting the most common CAD formats, such as IGES and STEP, as well as CATIA files, parametric meshing strategies, able to follow the shape changes based on the CST formulation, are implemented to automatically generate structural and aerodynamic models. It interacts with different classes and methods, as shown in Figure 2, to generate $3 \mathrm{D}$ models for different disciplines. During the shape optimization described in this paper, only aerodynamic analyses have been performed in order to evaluate the performances of wings with and without morphing devices. Therefore the generation of structural models, based on the OOP-based PFEM (Parametric Finite Element Models) class, as well as the postprocessing class including Fluid-Structure Interaction (FSI) techniques, is not described [22].

PHORMA is equipped with a CAD interface to automatically interpolate in spanwise direction the set of cross sections describing the wing. Corresponding parametric shapes are automatically transformed into spline-based curves by means of a user-defined number of points extracted from the CST formulation and combined to generate a multisection NURBS-based surface by sweeping them along a user-defined spine. In order to accurately describe the original geometry, one or more guide curves can be defined and slope and curvature boundary conditions can be introduced at the ends of the surface. Comparisons between original wing geometries and identified shapes are performed to identify the correct combination Bernstein polynomial order/number of sections in spanwise direction able to guarantee an approximation error tolerance.

The generation of aerodynamic models is strictly connected to the full parametric description of the geometry. Both $2 \mathrm{D}$ and $3 \mathrm{D}$ models can be produced by different inhouse procedure depending on the desired model quality.

In the case of $2 \mathrm{D}$ aerodynamic analyses, a specific code able to automatically produce a $2 \mathrm{D}$ structured mesh suitable to perform Navier-Stokes computations is directly linked to the CST-based code. The generation of structured mesh around a parameterized airfoil is based on a script for Ansys ICEMCFD [31]. It is represented by a Tcl/Tk shell with an extended library consisting of ICEMCFD commands.
The script is contained in a "rpl" file, while the complete process, shown in Figure 4, works by adapting the mesh with respect to a predefined mesh, contained in the "blk" file, built around a baseline airfoil shape. Once the aerodynamic mesh is produced, the CFD computations are performed by the EDGE code [32]. The parametric mesh generation has been applied to the morphing leading and trailing edge of the airfoil shown in Figure 5.

The whole process allows adding a set of high-fidelity polar curves to the set of the most important parameterized cross sections used by PHORMA to describe the complete wing model. This could be used to make local corrections to a 3D low-fidelity simulation with the computational cost of a reduced number of $2 \mathrm{D}$ flow solutions equal to the number of cross sections $[33,34]$.

In the case of $3 \mathrm{D}$ aerodynamic analyses, the cross sections are combined in order to spread the specific geometry details along the wing via particular interpolation surfaces able to accurately reproduce the correct wing thickness distribution in spanwise direction. The implemented procedure allows imposing different transition laws among different 2D airfoils, as well as modifying their properties such as Angle of Attack, dihedral or tow angles. Different kind of low-fidelity and high-fidelity aerodynamic models can be generated exploiting the parametric methods that PHORMA inherits from the CST formulation.

Another way to perform $3 \mathrm{D}$ simulations is to directly generate full volume meshes for medium/high-fidelity analyses. PHORMA is linked in batch mode to the software sumo $[35,36]$ in order to generate unstructured surface meshes. Heuristics parameters can be tuned to obtain acceptable mesh. Triangulations are based on a three-dimensional insphere criterion, which provide high quality meshes. Geometric refinement criteria are able to produce a finer mesh in regions of strong curvature, such as morphing leading edge geometries. Starting from the surface mesh, unstructured or hybrid volume meshes can be generated by TetGen code and automatically converted to EDGE-compatible files in order to perform Euler or Reynolds average Navier-Stokes formulation (RANS) computations.

The hybrid mesh generation has been applied to the Reference Aircraft. The prismatic layer around the fuselage and around a wing section placed near the root equipped with a morphing leading edge is shown in Figure 6. Once the aerodynamic analysis is finished, PHORMA is able to extract the results in terms of $C_{p}$ distribution and to apply them along corresponding parametrized cross section shapes. The aerodynamic results can be extrapolated around one or more sections arbitrarily positioned and oriented, so that the set of wing cross sections can be directly associated with a set of aerodynamic results coming from $3 \mathrm{D}$ analyses.

\section{Optimization Problem Definition}

The skin structural requirements, introduced in Section 3.3, are embedded into the implementation of the shape optimization problem as described in Sections 4.1 and 4.2, so that the aerodynamic performances are evaluated only on physically acceptable shapes. The process exploits parametric 


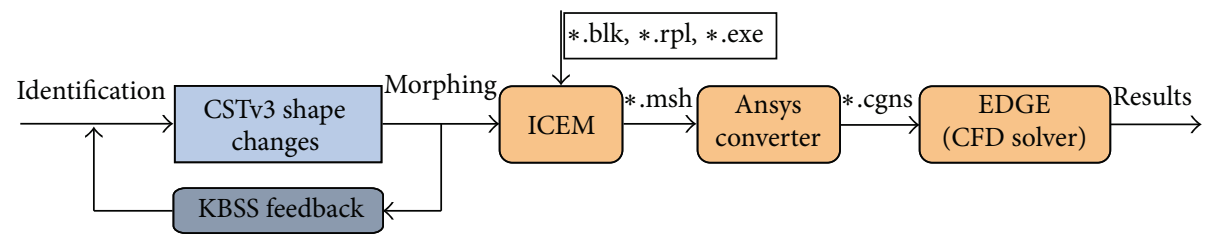

FIGURE 4: The implemented procedure for 2D CFD analyses embedded into the CST class.
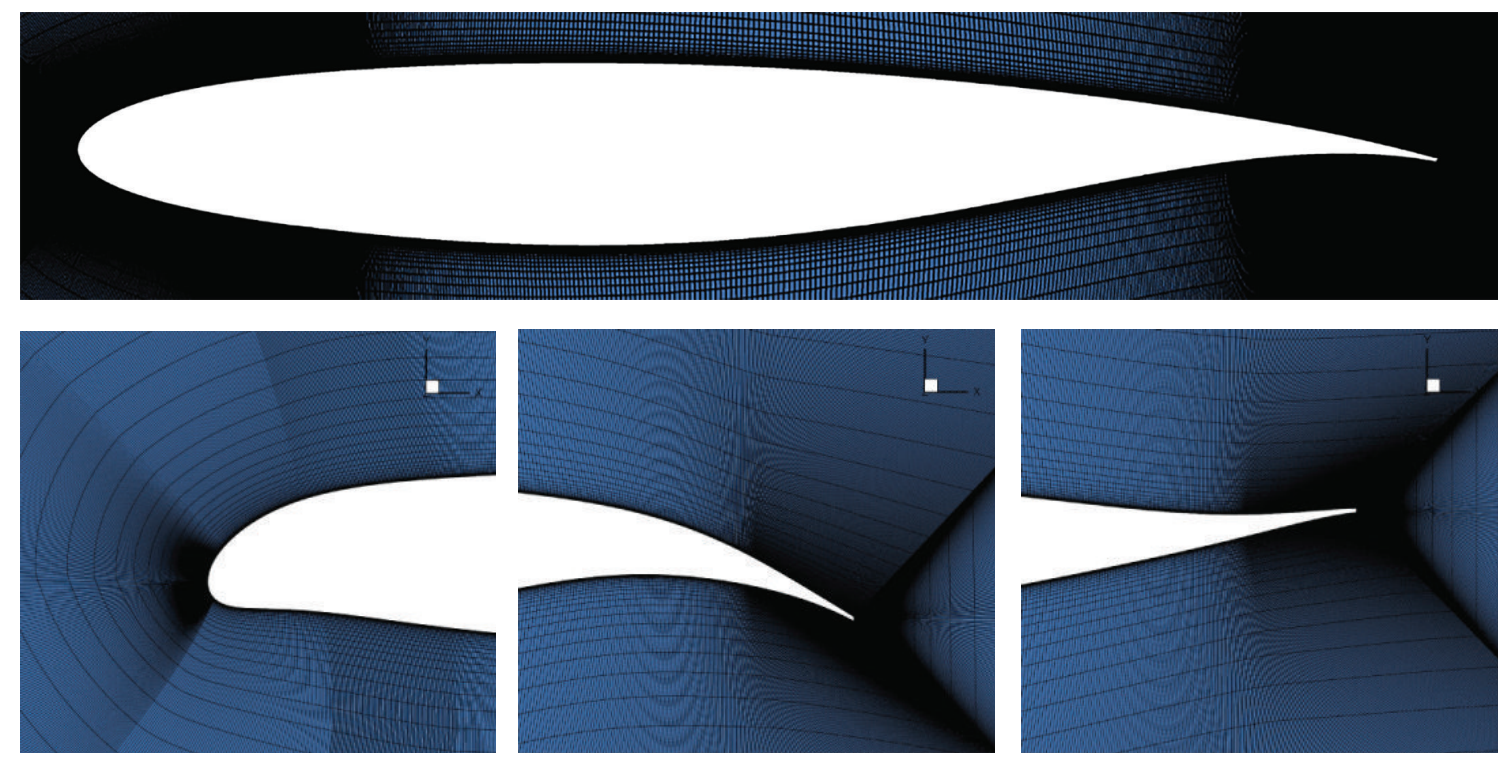

FIGURE 5: Parametric meshing around undeformed airfoil and corresponding morphing leading and trailing edge shapes.

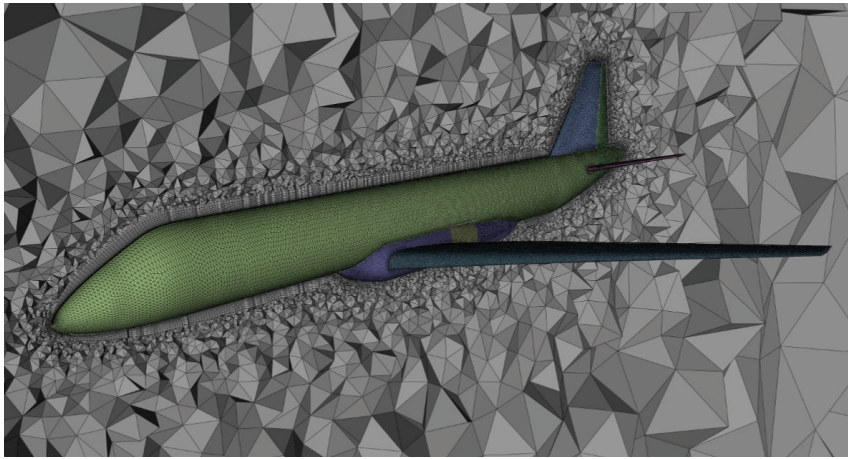

(a)

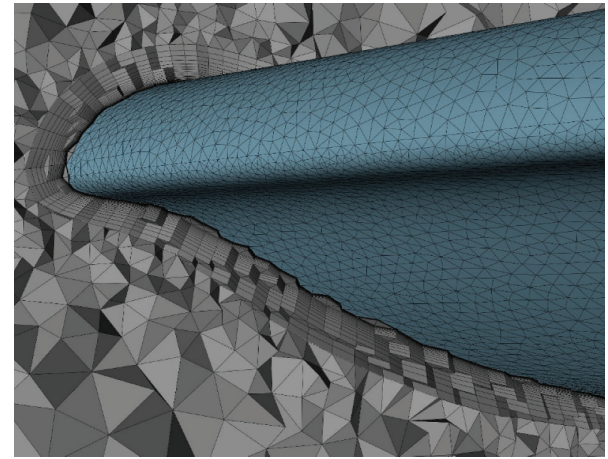

(b)

FIGURE 6: 3D hybrid mesh for RANS computation around complete aircraft w/o engine (a) and around the morphing leading edge (b).

meshing capabilities, described in Section 3.4, to generate the aerodynamic models. This aerostructural scheme allows combining the skin structural control and the estimation of global aerodynamic performances in the same shape parametric representation, and it is suitable to describe a wide variety of wing shapes using a small number of design variables.

4.1. Wing-Box Implicit Constraint. When a perturbation is introduced in the CST geometry by means of a subset of coefficients $A_{i}$, the shape changes spread on the entire domain and the other coefficients must be scaled in order to keep the shape fixed along specific regions defined by $\Delta \psi$ intervals. If the number of perturbed coefficients is $m$, the other $n-m+1$ coefficients can be quickly computed, starting from the following equation:

$$
\begin{aligned}
\mathbf{t}_{(n-m)}(\psi) \cdot \mathbf{a}^{T}= & \zeta(\psi)-\psi \zeta_{\mathrm{TE}}-(1-\psi) \zeta_{\mathrm{LE}} \\
& -C_{N 2}^{N 1}(\psi) \sum_{i=1}^{m} A_{i} B_{i}(\psi) .
\end{aligned}
$$


Given $\left\{\chi_{j}\right\}_{1 \leq j \leq l+1} \in\left(\Delta \psi_{1}, \ldots, \Delta \psi_{k}\right) \in(0,1)$ pairwise distinct real points, included in a number of fixed subregions equal to $k$, computing the $(n-m+1)$ coefficients $\mathbf{a}$, it is equivalent to solve the following reduced system, smaller than that reported in (6):

$$
\mathbf{T}_{(n-m)}^{T} \mathbf{T}_{(n-m)} \mathbf{a}^{T}=\mathbf{T}_{(n-m)}^{T} \mathbf{f}
$$

where $\mathbf{T}_{(n-m)}$ is here defined $(l+1) \times(n-m+1)$ reduced CST-Vandermonde matrix for the CST basis $T_{(n-m)}$ of degree less than or equal to $n-m$ and $\mathbf{f}$ is the vector obtained evaluating the right term of $(10)$ in the $(l+1)$ nodes. In this way the perturbation affects only the domain outside the fixed regions. The code automatically selects the $m$ coefficients, by the comparison between the subdomain and the positions of the airfoil components peaks, defined in (2).

This approach is very efficient for the following main reasons:

(i) It allows introducing local perturbations by means of a global geometry representation method.

(ii) It preserves the $C^{2}$ continuity and the local smoothness of the wing geometry, which is one of the most important features of the CST formulation.

(iii) It reduces the number of external variables, from $n$ to $m$.

(iv) It makes implicit constraints along the regions which must be kept undeformed.

The results of a simple 2D example are reported in Figure 7 to show the effects of the approach here presented. The grey area corresponds to the region, of a considered airfoil, that must be kept undeformed. The blue surface is the CST parametric representation of the upper surface corresponding to Bernstein Polynomials Order of 8 (BPO8). The green surface was obtained multiplying the coefficient $A_{2}$ by a factor equal to 3 . The red surface was obtained in the same way, but activating the solution of (10). It is possible to see how the perturbation spreads along the entire airfoil domain in the first case, while it is restricted outside the undeformed region in the second case.

The approach here presented is extended to three dimensions by the CAD interpolation capabilities described in Section 3.4. Figure 8 shows an example of shape perturbations introduced outside the wing-box area of a $3 \mathrm{D}$ wing.

This method allows implementing implicit wing-box constraints into the shape optimization. Every time, the $m$ coefficients of each section are changed and the least square problem is solved to constrain the shape in the undeformed region. Since the least square error does not guarantee that the shape passes through every surface point, the new shape is not exactly the same compared to the original one within the undeformed region. In 2D, the differences between the reference and the deformed airfoil rapidly vanish increasing the order of the Bernstein polynomial, following the same trend already described in [37]. In $3 \mathrm{D}$, these residual differences

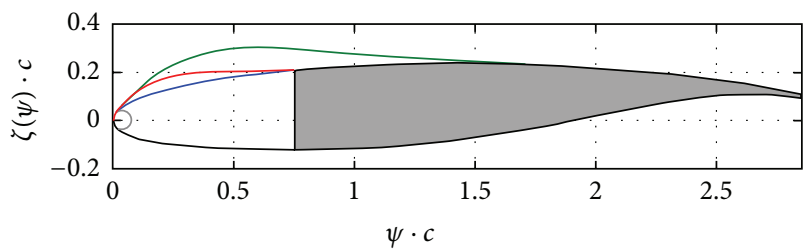

FIGURE 7: Comparison between a generic airfoil (black), its parameterization (blue), and corresponding shapes obtained introducing the same perturbation with (red) and without (green) solving the reduced system.

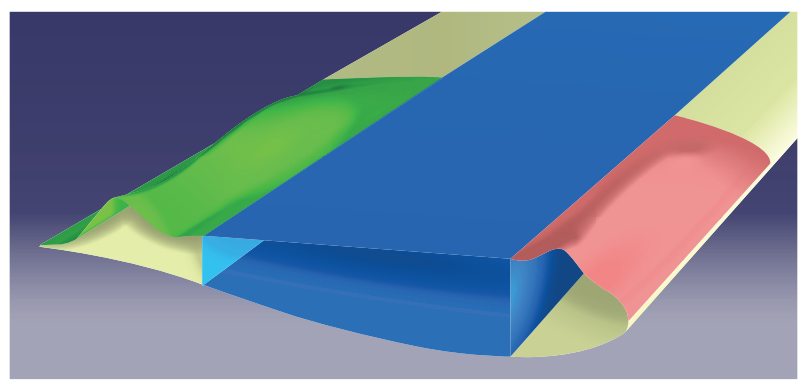

FIGURE 8: Local perturbation and fixed wing-box parametric representation in three dimensions.

also suffer the approximation introduced by the CAD-based interpolation technique working in the spanwise direction. The comparison between the actual and the deformed wing shape of the Reference Aircraft showed that using Bernstein Polynomial of Order eight and Bernstein Polynomial of Order ten to represent eight cross sections along the span is a good compromise that allows introducing acceptable spanwise deflection law in the morphing regions and, at the same time, obtaining a maximum error within the tolerance of $0.1 \mathrm{~mm}$ in the wing-box region. CFD analyses showed that the pressure distribution for a wing shape affected by this level of error appears to match the actual wing pressure distribution.

Wing-box constraints are very common in the aircraft shape design and several approaches that allow achieving this goal can be found in the literature. Different parameterization methods could be used to improve the approximation in the undeformed region [38] or to increase the efficiency when they are used within an optimization process [39]. However the estimation of the morphing skin structural behaviour is based on the use of Bernstein polynomial as shape function that allows the structural quantities of Section 3.3 to be analytically computed.

4.2. Morphing Skin Structural Requirements. Combining the estimation of the structural behavior of the morphing skin and the introduction of feasible perturbation described in Sections 3.3 and 4.1, the KBSS feedback represents a specific algorithm that allows introducing only morphing shape changes that meet preassigned structural requirements. The design variables are composed by a subset of $m$ independent 

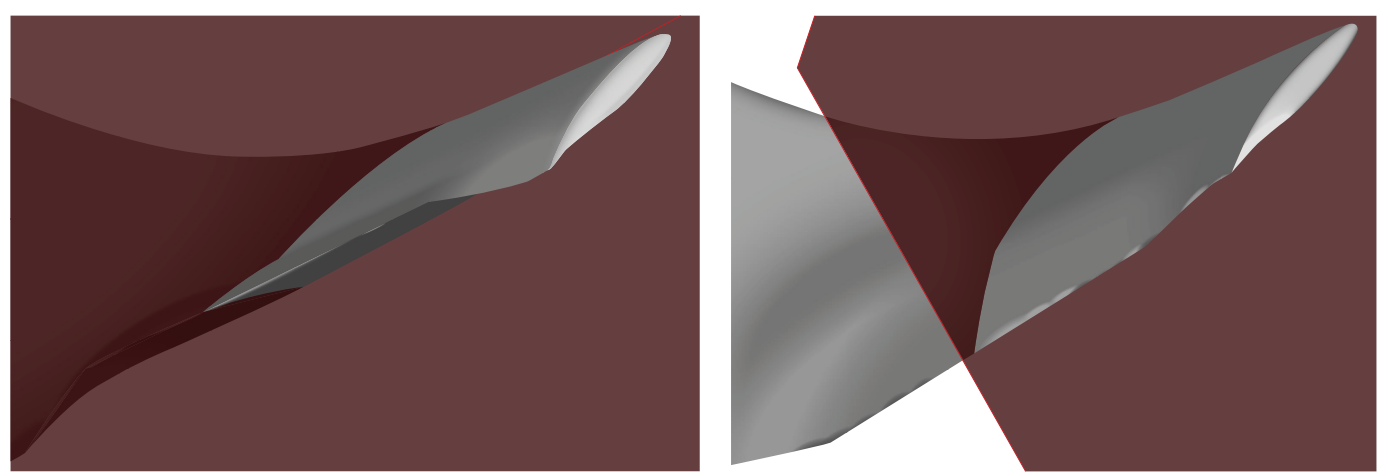

FIGURE 9: Parametric CAD model of a morphing winglet.

coefficients $A_{i}$, while the general form of the algorithm can be expressed as follows:

$$
\begin{array}{ll}
\text { Minimize } & w \cdot \varepsilon_{\text {axial }}(\text { nal })+(1-w) \cdot \varepsilon_{\text {bend }}(\text { nal }) \\
\text { such that } \quad & \mathbf{T}_{(n-m)}^{T} \mathbf{T}_{(n-m)} \mathbf{a}^{T}=\mathbf{T}_{(n-m)}^{T} \mathbf{f} \\
& \left|\Delta L_{\mathrm{dev}}\right| \leq k_{L} \cdot L_{\mathrm{dev}, u} \\
& |\Delta A| \leq k_{A} \cdot A_{u},
\end{array}
$$

where $w$ is a weight coefficient that allows scaling the axial and the bending strain component and represents a degree of freedom to obtain different morphing shapes. This expression depends on the particular case study and can be replaced with a more suitable form. It represents a sort of shape variation law which defines a set of "KBE parameters" that can be changed to provide a different control of the shape. Afterwards, the first term is the implicit constraint for the solution of (10). It acts on the structural box region delimited by the front and rear spar position and described by $l+1$ pairwise distinct coordinates $\left\{\chi_{j}\right\}_{1 \leq j \leq l+1} \in\left(C_{\mathrm{FS}}, C_{\mathrm{RS}}\right) \in\left(x_{\mathrm{LE}}, c\right)$. The second term $k_{L}$ sets the maximum length variation of the morphing device skin $\Delta L_{\mathrm{dev}}$, with respect to the undeformed one $\left(L_{\mathrm{dev}, u}\right)$. It can be related to the skin of a leading edge device $\left(\Delta L_{\mathrm{LE}, \text { skin }}\right)$ or to the upper and lower skin of a trailing edge device $\left(\Delta L_{\mathrm{TE}, \text { UpperSkin }}\right.$ and $\left.\Delta L_{\mathrm{TE} \text {,LowerSkin }}\right)$. The inflatable term $k_{A}$ that allows limiting the maximum allowable airfoil internal area variation $\Delta A$, with respect to the undeformed one $\left(A_{u}\right)$, is introduced to avoid the generation of unrealistic morphing shapes with too much stretched thickness. More terms, such as stress limits, can be added. It was noticed that quantities such as length and curvature, then strain, and stress depend on the actual size of the shape and are computed with respect to the variable $x$ instead of the nondimensional $\psi$.

Algorithm which implements (11) can be applied to each parametric shape included in the set of the most important sections used to describe the complete 3D aircraft. It works as a background process that provides a structural retrofit able to interactly change the morphing wing shape in a feasible way.

The simplest example to demonstrate how the KBSS feedback acts is to force the skin axial and bending deformation to be equal to zero that should be equivalent to a rigid rotation of a control surface. A rigid tab connected to a winglet by means of two morphing transition regions is shown in Figure 9. In this case the KBSS feedback worked to introduce two equivalent rotations of $10 \mathrm{deg}$ downward and $15 \mathrm{deg}$ upward over a deformable region which starts at $80 \%$ of the local chord.

4.3. Genetic Coordinates. When the shape changes are introduced by means of the shape optimization of Section 2, the KBE parameters are included between the optimization variables, in addition to the CST parameters $R_{\mathrm{LE}}, Z_{\mathrm{LE}}, \beta, \delta$, $Z_{\mathrm{TE}}, \Delta Z_{\mathrm{TE}}$, and $c$ defined in Section 3.1, and together with the $n-1$ shape function Bernstein polynomial extra coefficients for the upper surface and other $n-1$ for the lower surface.

According to the constant cross section length (CCL) concept [15], when the morphing leading or trailing edge changes its shape, the airfoil chord becomes smaller in order to keep the skin length constant or satisfy the structural constraints. The CST formulation, as well as the aerodynamic computations, works on nondimensional equation, but all the structural quantities, such as $\varepsilon_{\text {bend }}, \Delta \kappa(x)$, and $\Delta L_{\mathrm{dev}}$, are dimensional and depend on the actual size of the morphing skin.

Once the wing-box region is detected, a subset of CST parameters can be defined, as described in Section 4.1. If a morphing leading edge is considered, for example, only $Z_{\mathrm{LE}}$ is considered a design variable used by the highlevel optimization which runs the aerodynamic computation, while the other CST parameters are fixed by the boundary conditions. On the other side, the KBSS feedback works on a subset of $m$ extra coefficients, in addition to $R_{\mathrm{LE}}$ and the airfoil chord $c$, that represent implicit variables, not included in the set of the high-level optimization variables and allow satisfying the structural constraints included in the low-level optimization loop (11).

Both crossover and mutation subroutines execute the KBSS feedback in order to preserve the local feasibility, while dedicated subroutine allows mixing only the same type of genetic coordinates. The crossover randomly selects a pair of parents who produce a new pair of offsprings by switching information about a user-defined number of CST parameters of the same cross section, preserving the KBE parameters. The effect is to mix CST parameters between different individuals 

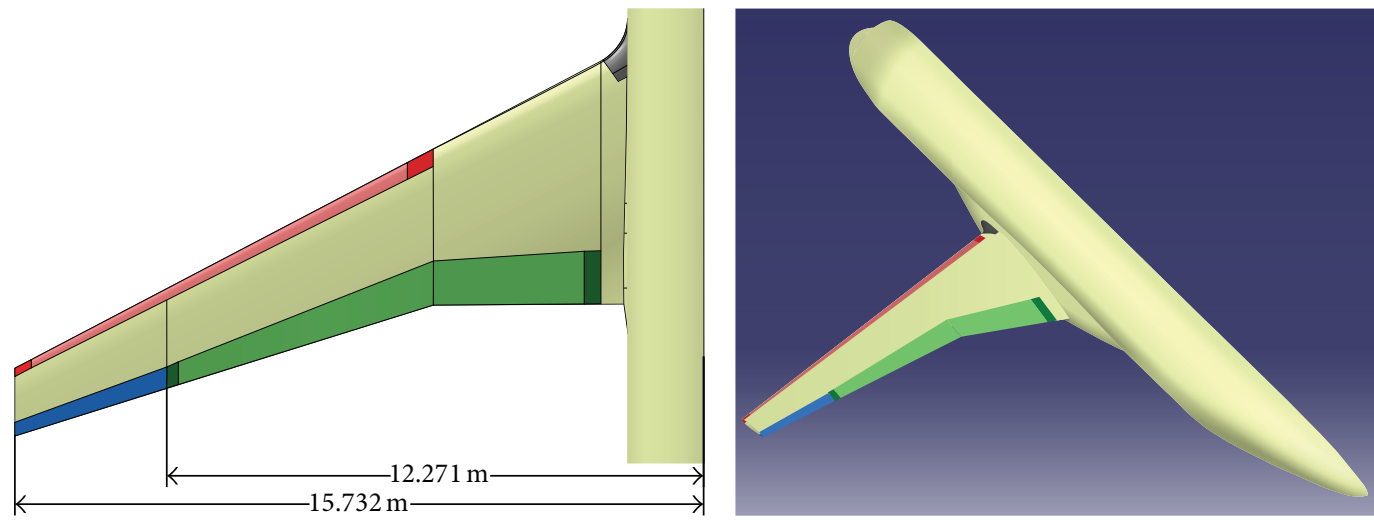

FIGURE 10: Reference Aircraft with morphing wing configuration and corresponding wing planform.

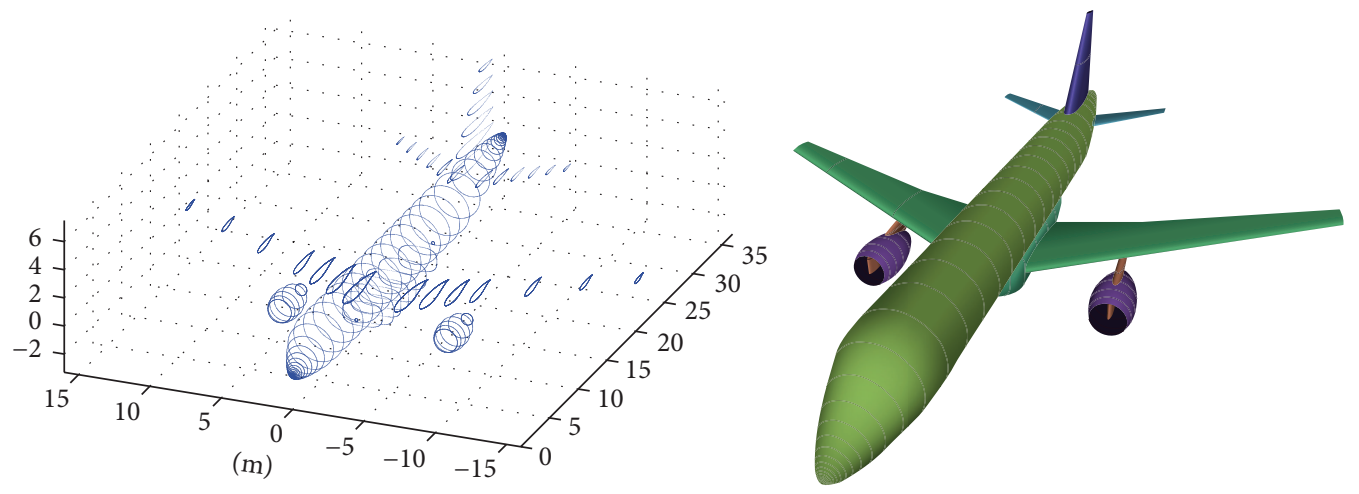

FIgURE 11: Parametric identification applied to the Reference Aircraft model.

and KBE parameters between different cross sections. The mutation can directly change a user-defined number of KBE or CST parameter values in order to modify the local shape.

\section{Results}

The procedure described in the previous sections has been applied for the optimization of the so-called Reference Aircraft (RA). The morphing wing configuration and some information about the wing planform, such as the total wing span and the aileron span position, are shown in Figure 10. The RA is a typical regional jet capable of carrying 113 PAX which provides operational flexibility to accomplish different missions at the transonic regime. It is capable of missions that encompass $600 \mathrm{~nm}$ up to $2300 \mathrm{~nm}$. The Maximum Takeoff Weight is $58000 \mathrm{~kg}$ and the Maximum Landing Weight $53000 \mathrm{~kg}$, while the Maximum Payload and the Maximum Fuel are, respectively, $14000 \mathrm{~kg}$ and $18000 \mathrm{~kg}$. The Reference Aircraft was designed to achieve an optimum cruise performance at Mach number equal to 0.78 , an altitude of $37000 \mathrm{ft}$, and an Angle of Attack $A o A$ of $2 \mathrm{deg}$, corresponding to a lift coefficient $C_{L}$ of 0.47 . The landing condition corresponds to an Angle of Attack $A o A$ of $15 \mathrm{deg}$, while the considered Landing Mach is 0.15 .

Different sections were chosen in order to identify the parametric shape of the Reference Aircraft and to build up the mathematical model suitable to be used to introduce the shape changes into the leading and trailing edge morphing regions. The whole process, described in Section 2, has been applied to the parametric model of the Reference Aircraft which is shown in Figure 11, together with the identified cross sections.

In the following subsections, some results for morphing leading and trailing edge at different flight conditions are summarized. Since different design requirements have been provided for each morphing device, different shape optimization problems have been defined. They represent four aerodynamic shape optimizations including structural constraints on the morphing skin. According to the approach described in Section 2, they allow collecting a family of optimal morphing shapes that can be adopted as multiple targets for the design of a morphing mechanism.

\subsection{D Shape Optimization of the Morphing Leading Edge for} Cruise Condition. At the beginning, a 2D shape optimization based on RANS computations has been applied to the wing section placed at $9 \mathrm{~m}$ along the wing span, in the reference system of Figure 10, to lead the shape design of a morphing leading edge. After the corresponding airfoil was identified, a simplified version of the procedure described in Section 2 has been combined to the scheme shown in Figure 4 for the evaluation of the $2 \mathrm{D}$ aerodynamic coefficients. In this way, 
the process automatically produces the mesh of both clean and morphing airfoil shown in Figure 5, in order to estimate the maximum benefit in terms of minimum $C_{d}$ keeping the $C_{l}$ as constant as possible. In order to guarantee the trim condition, this last constraint forces the lift coefficient to be, if not equal, at least greater than the lift coefficient of the reference airfoil. The evaluation of the aerodynamic coefficients is performed at the cruise condition of the RA. The optimization problem is formulated as follows:

$$
\begin{array}{ll}
\text { Minimize } & C_{d} \\
\text { such that } & \mathbf{T}_{8}^{T} \mathbf{T}_{8} \mathbf{a}^{T}=\mathbf{T}_{8}^{T} \mathbf{f} \\
& \Delta \psi_{\text {wing-box }} \equiv(0.1,1 .) \\
& C_{l} \geq 0.566 \\
& |\Delta A| \leq 0.15 \cdot A_{u} \\
& \left|\Delta L_{\mathrm{LE}, \text { skin }}\right|=0 . \\
& \max \left(\sigma_{\text {bend }}\right) \leq 215 \mathrm{MPa},
\end{array}
$$

where the first two constraints represent the equation system (10) used to keep undeformed region from the $10 \%$ of the chord up to the end of the trailing edge. The main design rule from a structural point of view is keeping the circumferential skin length $L_{\mathrm{LE} \text {,skin }}$ constant to avoid axial strains. The amount of droop of the individual section depends on the maximum bending stress into the skin $\left(\sigma_{\text {bend }}\right)$. The maximum value of stress is related to a conventional leading edge skin made in aluminium alloy $(E=72 \mathrm{GPa})$ with a minimum thickness $t$ of $0.5 \mathrm{~mm}$ and to the curvature change which is restricted to $12(1 / \mathrm{m})$.

The parametric airfoil shape is described by Bernstein Polynomials Order $n=10$ (BPO10) that involves a total of 22 extra coefficients, 11 for the upper skin and 11 for the lower one. Nevertheless, the optimization variables are composed of $1 \mathrm{CST}$ parameter $Z_{\mathrm{LE}}$ and $1 \mathrm{KBE}$ parameter $w$. The dimension of each genetic array is equal to 2 , while the KBSS feedback works on a total of $m=6$ implicit variables, 2 for the upper surface $\left(A_{\text {up }, 1}\right.$ and $\left.A_{\text {up }, 2}\right), 2$ for the lower surface $\left(A_{\text {low, } 1}\right.$ and $\left.A_{\text {low, } 2}\right)$, in addition to $R_{\mathrm{LE}}$ and to the airfoil chord $c$, as described in Section 4.3. The extra coefficients $A_{1}$ and $A_{2}$ correspond to the shape component peaks placed in the region of the morphing leading edge. The genetic algorithm started with an initial population of 15 individuals and allows 50 generations. The CFD grid is composed of 260452 nodes; each individual runs in parallel on 1 dedicated quad-core CPU, with processor base frequency of $2.93 \mathrm{GHz}$ and hyperthreading for a total of 8 threads, and requires about 20 minutes to solve RANS equations. On the other side, the KBSS feedback works for about 2 minutes, mainly due to satisfying the circumferential skin length constraint. The optimal solution was obtained after 5 days and 25 iterations. The total computational time could be drastically reduced using Parallel Genetic Algorithm (PGA).

The vertical deflection which results from the shape optimization is equal to $Z_{\mathrm{LE}}=-0.0260 \mathrm{~m}$ over an airfoil chord of $2.8486 \mathrm{~m}$, corresponding to an equivalent leading edge deflection $\delta_{\mathrm{LE}}=5.29 \mathrm{deg}$. The circumferential skin length constraint associated with this morphing deflection is satisfied, considering a numerical tolerance of $1 \cdot e^{-8}$. Corresponding benefits are computed by 2D RANS analyses in terms of aerodynamic coefficients that change from $C_{l}=$ $0.566, C_{d}=0.0185$ to $C_{l}=0.569, C_{d}=0.0175$, for a total efficiency improvement of $6.2 \%$. These results show that the considered morphing leading edge is able to efficiently change its aerodynamic shape without generating axial stresses. Figure 12 shows how the shape changes act on the fore region of the shock wave, adapting the aerodynamic field around the airfoil to increase the lift over drag ratio and to keep the lift coefficient constant or greater than the reference one. The optimal deformed airfoil shape is able to decrease the $C_{d}$ value, while the $C_{p}$ values increase along the upper skin and decrease along the lower skin so that the $C_{l}$ chordwise balance is similar to the undeformed one.

\subsection{D Shape Optimization of the Morphing Leading Edge} for Cruise Condition. The complete procedure described in Section 2 was applied to the $3 \mathrm{D}$ wing of the RA to maximise the improving of the aerodynamic efficiency $L / D$ of the wing in cruise condition by means of a morphing leading edge. A set of 7 parametric sections along the span, 4 equally spaced in the inboard region, from the wing root to the wing kink, and 4 equally spaced in the outboard region, from the kink to the wing tip, was identified in order to introduce the morphing shape changes. Each wing section is represented by Bernstein Polynomials Order $n=10$ (BPO10) for a total number of extra coefficients equal to 154 . The optimization variables are composed of $1 \mathrm{CST}$ parameter $Z_{\mathrm{LE}}$ and $1 \mathrm{KBE}$ parameter $w$ for each section, for a total of 14 explicit optimization variables that form each genetic array. Since the algorithm is applied to a $10 \%$ chordwise configuration, $7 \mathrm{KBSS}$ feedback works on 6 implicit variables $\left(A_{1}\right.$ and $A_{2}$ for the upper and lower surfaces, $R_{\mathrm{LE}}$ and $c$ ) for each parametric section. The optimization problem can be formulated as follows:

$$
\begin{array}{ll}
\text { Maximize } & L / D \\
\text { such that } & \mathbf{T}_{8}^{T} \mathbf{T}_{8} \mathbf{a}^{T}=\mathbf{T}_{8}^{T} \mathbf{f} \\
& \Delta \psi_{\text {wing-box }} \equiv(0.1,1 .) \\
& |\Delta A| \leq 0.12 \cdot A_{u} \\
& \left|\Delta L_{\text {LE,skin }}\right|=0 . \\
& \max \left(\sigma_{\text {bend }}\right) \leq 215 \mathrm{MPa} .
\end{array}
$$

The KBSS feedback acting on each wing section works to keep undeformed local regions between the $10 \%$ of the chord and the end of the trailing edge, to limit the airfoil area variation and to avoid the circumferential skin length variation. The optimization parameters are set up to $k_{L, \mathrm{LE}}=1 \cdot e-6$ $\left(\sigma_{\text {axial }}=0\right)$ and $k_{A}=0.12$. The maximum deflection range is defined by the same curvature constraint used in the $2 \mathrm{D}$ optimization, while a 3D constraint was added in order to keep linear deflection law along the wing span. 

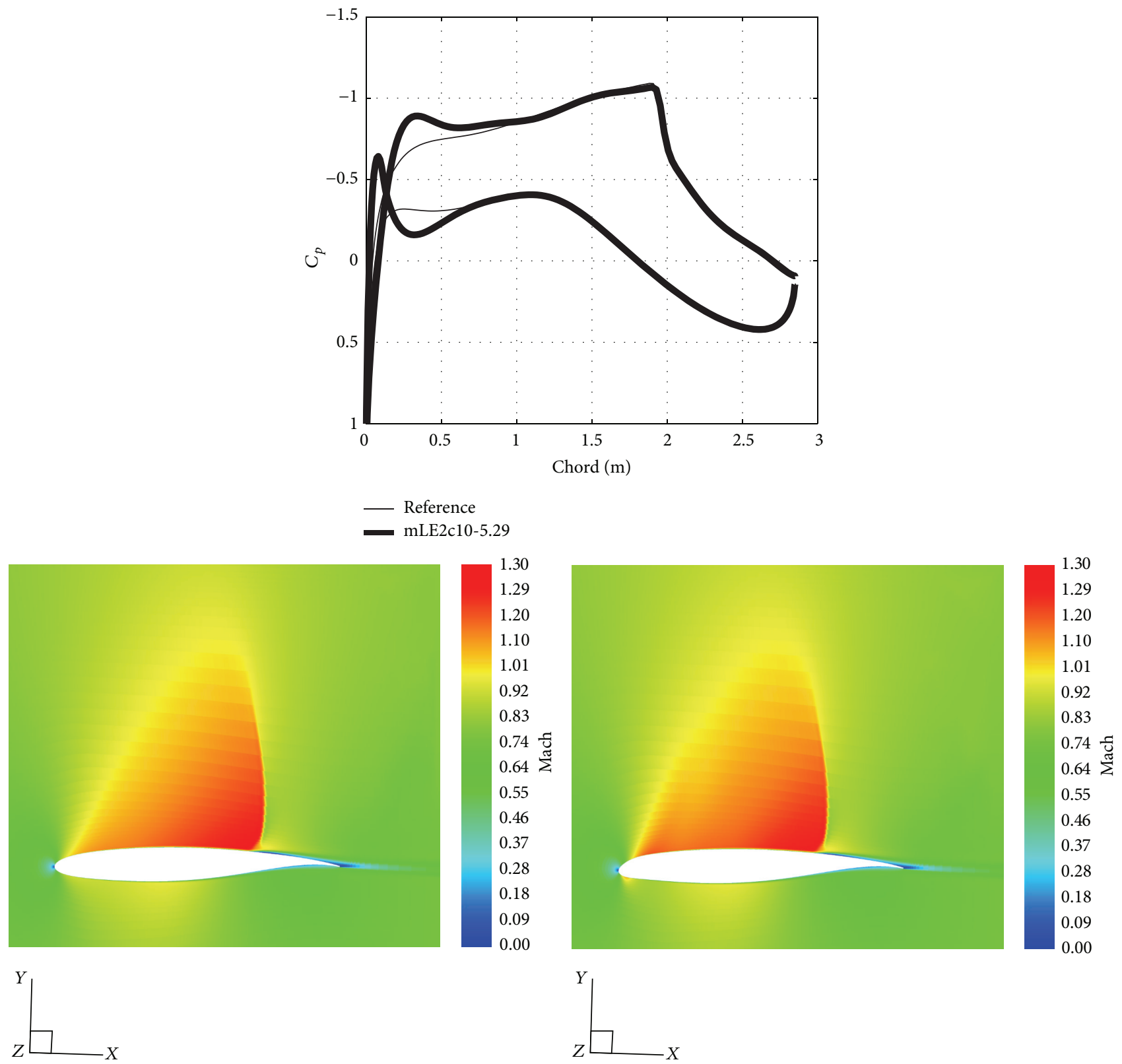

FIGURE 12: Morphing leading edge in high speed conditions: comparison between reference and deformed shape $2 \mathrm{D}$ results in terms of $C_{p}$ and Mach chordwise distributions.

The genetic algorithm started with an initial population of 40 individuals and allows 100 generations. The CFD grid is composed of 1. million nodes; each individual runs in parallel on 1 dedicated quad-core CPU with hyperthreading, for a total of 8 threads, and requires about 15 minutes of computational time to solve Euler equations. Each KBSS feedback works for 2 minutes on different threads. The optimal solution was obtained after about 2 weeks and 40 iterations.

The shape optimization process provided a spanwise distribution of optimal leading edge deflections $\delta_{\mathrm{LE}}$ that linearly decreases from $8 \mathrm{deg}$, at the wing root, to $7.2 \mathrm{deg}$, at the station placed at the $25 \%$ of the outboard region.
Beyond morphing shape variations vanish and no deflections are introduced by the optimizer from $25 \%$ to $100 \%$ of the outboard region.

The results are reported in Figure 13 and show how the leading edge shape changes are able to adapt the aerodynamic field over the wing in order to increase the wing efficiency. The black line represents the iso-Mach curve corresponding to a local velocity equal to the speed of sound. The results show that, in the transonic condition, the morphing leading edge is able to change the chordwise $C_{p}$ distribution inside the shock wave, following the same trend of Section 5.1. Figure 14 shows the chordwise $C_{p}$ distribution around the wing section placed at $4.7 \mathrm{~m}$ along the wing span in the reference system 


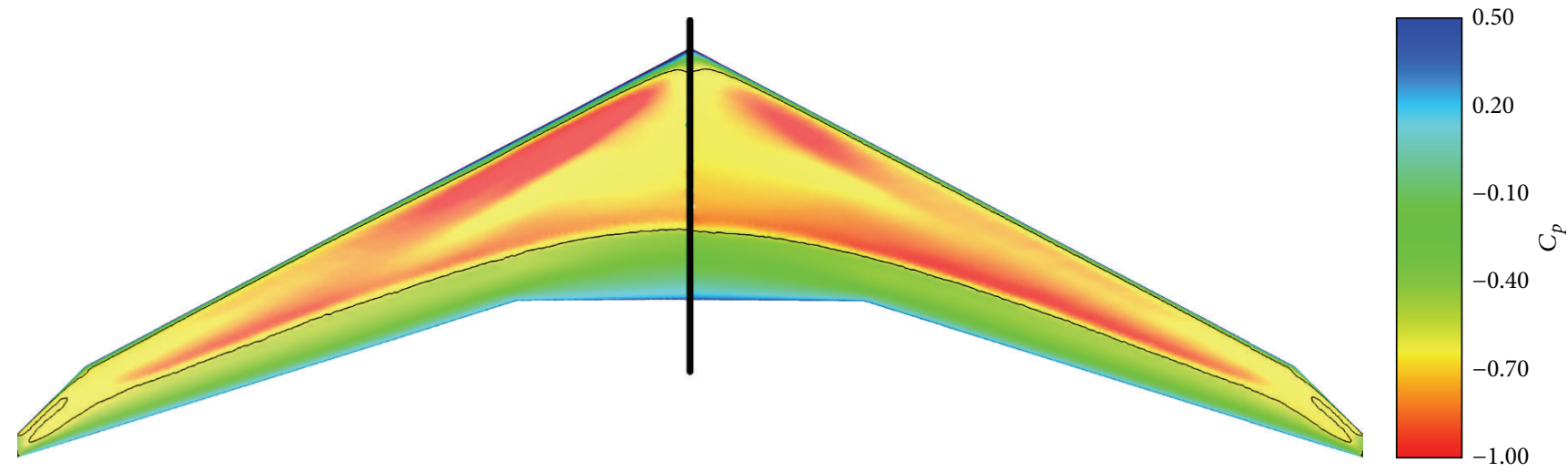

FIgURE 13: Comparison between the $C_{p}$ distributions at cruise Mach over the reference wing (right) and over the wing equipped with morphing leading edge (left).

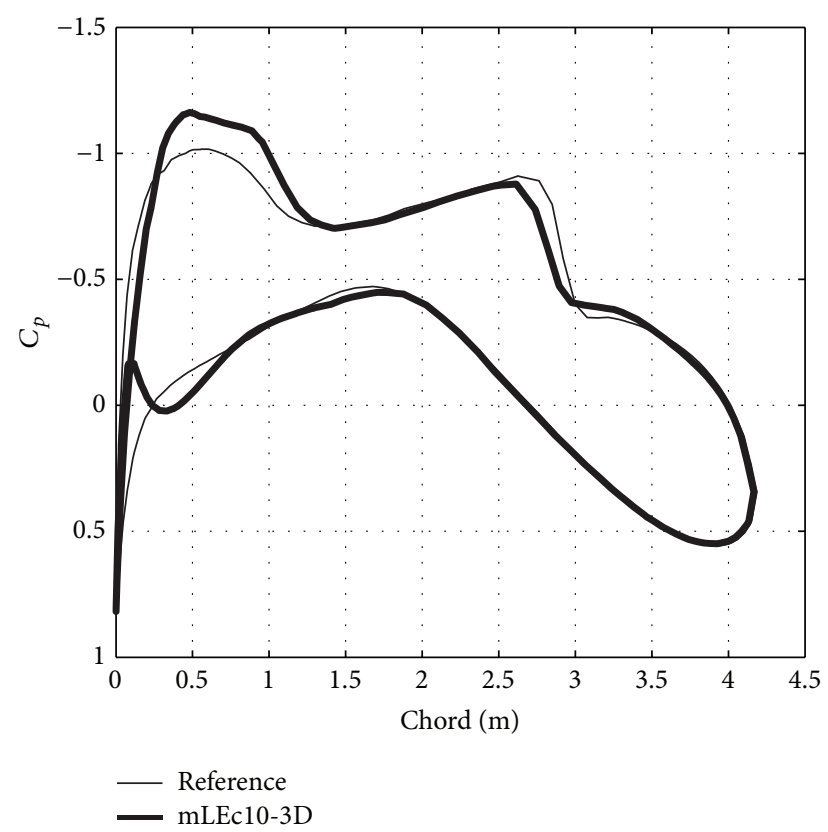

FIGURE 14: Comparison between reference and morphing shape 3D results in terms of $C_{p}$ chordwise distribution of the wing section placed at $4.7 \mathrm{~m}$ along the wing span.

of Figure 10. However since the swept wing reduces the local speed, it is more difficult to achieve the improvements obtained in the 2D case and the corresponding increase of wing efficiency is limited to $3.4 \%$. For this reason the shape optimization works to introduce leading edge deflections only in the inboard region, where the local aerodynamic flow is still parallel to the asymptotic speed and more similar to the $2 \mathrm{D}$ case.

The optimization algorithm acted to decrease the reference lift coefficient from $C_{L}=0.49$, computed by Euler method, to $C_{L}=0.487$. This means that the Angle of Attack should be increased from 2 deg to 2.03 deg to guarantee the trim in cruise condition. Additional computations showed that this small variation does not reduce significantly the benefits related to this morphing shape solution.
5.3. 3D Shape Optimization of the Morphing Leading Edge for Low Speed Condition. A second 3D optimization process was run using an objective function that tries to introduce the maximum droop deflection along the wing span, instead of an aerodynamic performance estimation. This may be suitable for a low speed condition, corresponding to the landing condition of the RA. A set of 4 identified parametric sections, equally spaced along the outboard region, were used in order to introduce the morphing shape changes. The droop deflection to be maximized along the wing span is the equivalent leading edge deflection $\delta_{\mathrm{LE}}$ introduced in Section 3.1. Each wing section is represented by Bernstein Polynomials Order of $n=10$ (BPO10) for a total number of extra coefficients equal to 88 , and a family of possible shapes has been generated for different values of $w$. Each of the 4 KBSS feedback algorithms works on 6 implicit variables ( $A_{1}$ and $A_{2}$ for the upper and lower surfaces, $R_{\mathrm{LE}}$ and $c$ ) for each section when the algorithm is applied to the $10 \%$ chordwise configuration and 8 implicit variables $\left(A_{1}, A_{2}\right.$, $A_{3}, R_{\mathrm{LE}}$, and $c$ ) when the $20 \%$ chordwise configuration is considered. The total number of optimization variables is 4 explicit variables and 24 or 32 implicit variables depending on the type of chordwise morphing configuration. The optimization problem, for the $20 \%$ chordwise configuration, can be formulated as follows:

$$
\begin{array}{ll}
\text { Maximize } & \frac{1}{4} \sum_{i=1}^{4} \delta_{\mathrm{LE}, i} \\
\text { such that } & \mathbf{T}_{7}^{T} \mathbf{T}_{7} \mathbf{a}^{T}=\mathbf{T}_{7}^{T} \mathbf{f} \\
& \Delta \psi_{\text {wing-box }} \equiv(0.2,1 .) \\
& |\Delta A| \leq 0.2 \cdot A_{u} \\
& \left|\Delta L_{\mathrm{LE}, \text { skin }}\right|=0 . \\
& \max (\Delta \kappa(x)) \leq 28 \frac{1}{m} .
\end{array}
$$

The optimization works keeping the KBE parameter $w$ fixed on each section. It is considered as an external parameter in addition to the KBSS feedback ones. The constraints 
include a linear deflection law in spanwise direction along the outboard region, while the maximum skin curvature change is set to avoid strains above $1 \%$, according to what observed in other works, such as that described in [4]. Due to the high deflection levels, the curvature constraint is expressed in terms of curvature variation that depends only on geometrical considerations, instead of bending stress, that requires knowing the material properties and the skin thickness. The first one is not easy to estimate at this stage due to the specific materials used for the morphing skin; the skin thickness could be variable along the leading edge arc length. The optimal shapes are chosen according to the following consideration: the alleviation of the bending stress inside the skin corresponds to the increase of the leading edge radius that helps to preserve laminar flow for low speed and high lift conditions.

The genetic algorithm started with an initial population of 20 individuals and allows 80 generations. Since no CFD analysis was performed, the optimization process was very fast and the optimal solution was obtained after less than 1 day, only to satisfy all the structural constraints along the wing span. The shape optimization provided the morphing leading edge shapes shown in Figure 15. The maximum droop deflection, corresponding to the $20 \%$ chordwise configuration, linearly decreases from $\delta_{\mathrm{LE}}=18.3 \mathrm{deg}$ at the wing kink to $\delta_{\mathrm{LE}}=11.2 \mathrm{deg}$ at the wing tip. Corresponding curvature difference functions computed along the morphing leading edge arc length and along the wing span are reported in Figure 16 and show the values are lower than the maximum curvature change limit.

Different types of $10 \%$ and $20 \%$ chordwise morphing leading edge shapes for different values of $w$ were analyzed. Once the best shapes were selected, they have been implemented on the Reference Aircraft 3D model of Figure 11 and postprocessed adopting RANS computations. The analyses performed after the optimization process showed that, for the subsonic flight regime, there is an improvement in the lift over drag ratio between 0.43 and 1.0 of the lift coefficient [40]. This improvement is about $5 \%$ at $C_{L}=0.6$ for the $10 \%$ chordwise configuration and the same value can be achieved at $0.8 \leq C_{L} \leq 0.9$ for the $20 \%$ chordwise configuration. In order to quantify the benefits in terms of fuel saving a typical flight mission was considered. This mission comprises $600 \mathrm{~nm}$, point to point distance, with a holding phase of 45 minutes. It was noticed that the holding phase is performed at the lift coefficient that falls inside the range in which the $10 \%$ chordwise configuration can provide benefits in the $L / D$ ratio. The adoption of this kind of morphing device at this flight phase would lead to $62 \mathrm{Kg}$ of fuel saving.

5.4. 3D Shape Optimization of the Morphing Trailing Edge for Cruise Condition. The shape optimization procedure was applied to the wing of the Reference Aircraft to improve the performances by means of a morphing trailing edge optimized for the cruise condition. The fitness function is related to the aerodynamic efficiency $L / D$, while the optimization process works without spanwise deflection law constraints and tries to keep the total $C_{L}$ not less than the reference one requested for trimming. A set of 7 parametric sections along the span, 4 equally spaced in the inboard region from the wing root to the wing kink section and 4 equally spaced in the outboard region from the same kink section to the transition region before the aileron, was identified in order to introduce the morphing shape changes. Each wing section is represented by Bernstein Polynomials Order of $n=8$ (BPO8) for a total number of extra coefficients equal to 126 . The optimization variables are composed of 2 CST parameters $\beta, Z_{\mathrm{TE}}$, and $1 \mathrm{KBE}$ parameter $w$ for each section, for a total of 21 explicit optimization variables that form the genetic array. Since the algorithm is applied to a $28 \%$ chordwise configuration, each of the $7 \mathrm{KBSS}$ feedback algorithms works on 7 implicit variables $\left(A_{5}, A_{6}\right.$, and $A_{7}$ for the upper and lower surfaces, in addition to the airfoil chord $c$ ), for a total of 49 implicit variables. The optimization problem can be formulated as follows:

$$
\begin{array}{ll}
\text { Maximize } & L / D \\
\text { such that } & \mathbf{T}_{5}^{T} \mathbf{T}_{5} \mathbf{a}^{T}=\mathbf{T}_{5}^{T} \mathbf{f} \\
& \Delta \psi_{\text {wing-box }} \equiv(0 ., 0.72) \\
& C_{l} \geq 0.49 \\
& |\Delta A| \leq 0.3 \cdot A_{u} \\
& \left|\Delta L_{\mathrm{TE}, \text { UpperSkin }}\right|=0 . \\
& \left|\Delta L_{\mathrm{TE}, \text { LowerSkin }}\right| \leq 0.05 \cdot c \\
& -5 \mathrm{deg} \leq \delta_{\mathrm{TE}} \leq+5 \mathrm{deg} .
\end{array}
$$

Different structural requirements were adopted for the trailing edge skin: the upper skin length constraint is set up to be constant $\left(k_{L, \text { UpperSkin }}=1 \cdot e-6\right.$ meaning that the upper skin axial strain is $\varepsilon_{\text {axial,UpperSkin }}=0$ ), while the lower skin length constraint has been relaxed in order to obtain an efficient aerodynamic shape. This means that the lower skin is free to slide along its contour and corresponds to the introduction of a linear slider to avoid axial strain. The constraints related to the skin curvature variations are not taken into account due to the small deflections which are bounded in the range of $\pm 5 \mathrm{deg}$. The inflatable term is set up to $k_{A}=0.3$ and the trailing edge equivalent deflection $\delta_{\mathrm{TE}}$ is bounded between $-5^{\circ}$ and $+5^{\circ}$.

The population size and the grid size of the aerodynamic model are more or less the same used for the study described in Section 5.2. The total computational time was about 10 days. The shape optimization provided the morphing trailing edge shapes shown in Figure 17. Corresponding optimal spanwise deflections increase from $-3.1 \mathrm{deg}$ at the wing root to $2.2 \mathrm{deg}$ at the transition region close to the aileron.

The aerodynamic results are reported in Figure 18 and show how the morphing trailing edge is able to change its shape in order to adapt the aerodynamic field around the wing. Differently from what happens for the morphing leading edge, the trailing edge deflection is able to move the shock wave, as shown by the black line that represents the iso-Mach curve where the local velocity is equal to the speed of sound. The trailing edge spanwise deflection moves the 

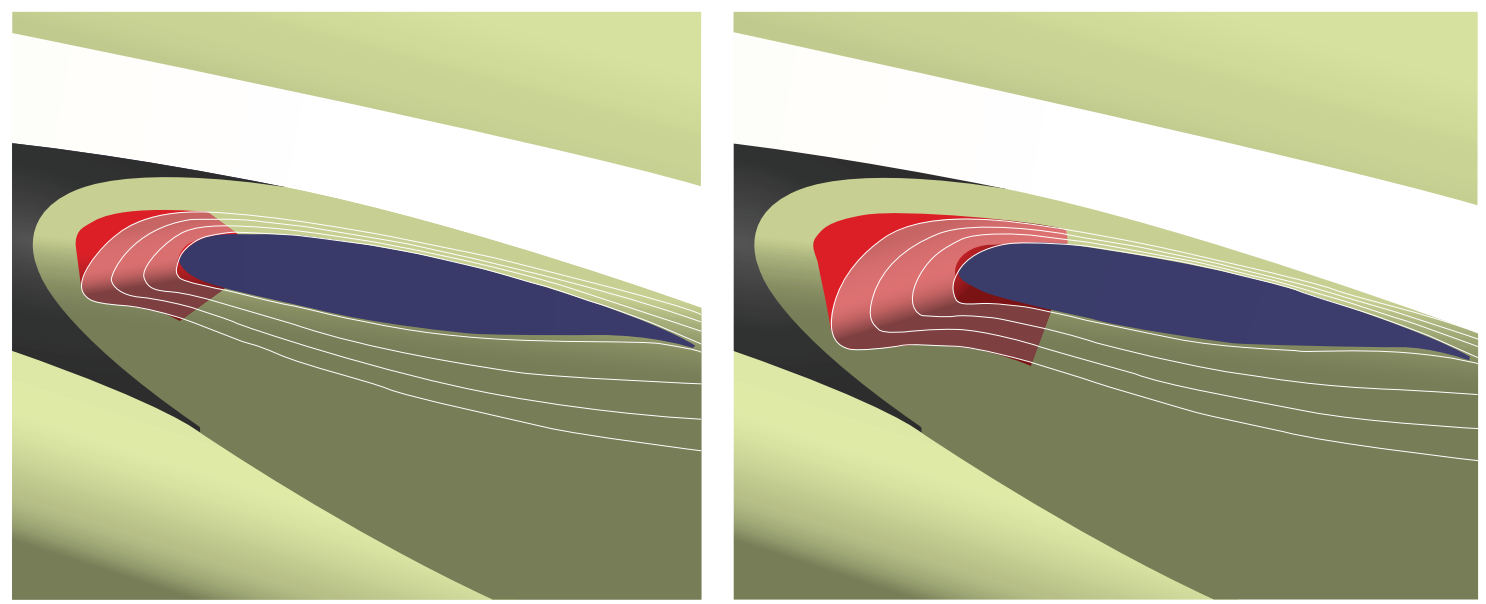

FIGURE 15: Parametric CAD model of the $10 \%$ and $20 \%$ chordwise morphing leading edge for maximum droop angle under skin structural constraint.
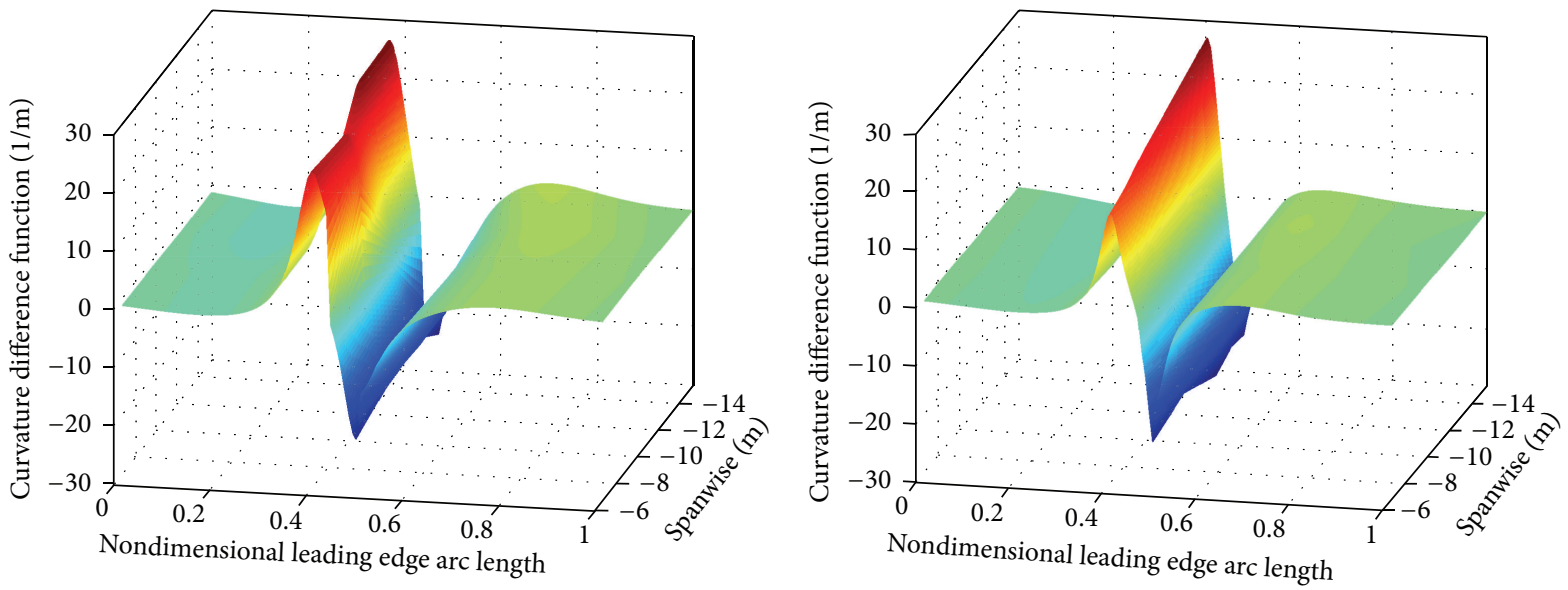

FIGURE 16: Curvature difference functions along the wing span for $10 \%$ and $20 \%$ chordwise maximum droop angle.

shock wave forward in the inboard region and backwards in the outboard region. In order to improve the wing efficiency, the spanwise antisymmetric deflections reduce the local lift coefficient $C_{l}$ in the region close to the wing root and increase it in the region near the aileron. This variation can be partially attributed to the wing load distribution which becomes elliptical rather than triangular. This effect, positive from the aerodynamic point of viewmoves the centre of pressure outboard and leads to increase of the root bending moment.

The optimization algorithm was able to improve the lift over drag ratio of $3 \%$, increasing the lift coefficient of $2 \%$. The trim condition should be recomputed and corresponding Angle of Attack reduced to obtain the same total lift coefficient required for the cruise. For this reason the morphing trailing edge shapes obtained by the optimization process have been implemented on the Reference Aircraft and several high-fidelity CFD computations have been performed on the complete aircraft [40]. The above decreasing deflection law in spanwise direction allowed increasing the $L / D$ of $2 \%$ for $C_{L}=$ 0.45 and $5.85 \%$ for $C_{L}=0.55$. This result is an indication that the potential aerodynamic benefits can be translated into fuel saving. However, the observed aerodynamic advantages are just part of the feasibility evaluation, which must involve a larger amount of disciplines, so during the structural design of the wing-box the impact on the overall wing weight of increasing the wing bending moment during the cruise condition should be evaluated.

\section{Conclusions}

Morphing seems a potentially promising technology allowing matching the new stringent requirements in terms of environmental impact of next generation aircraft. Unfortunately the results available in the literature concerning the application of morphing concepts still combine lights and shadows in terms or real benefits and open issues like certification, fatigue, and so on. For sure the lack of design procedures specifically dedicated to the optimal design of morphing mechanisms appears clearly. This paper introduced in a general way the work carried out at Politecnico di Milano to set up a complete framework for the optimal design 

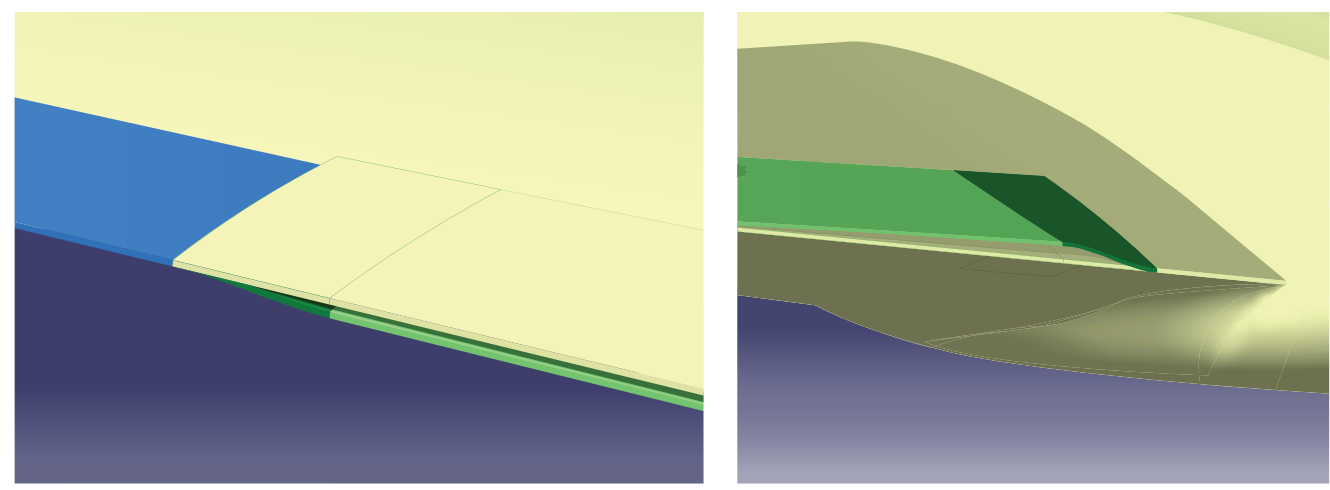

FIGURE 17: Parametric CAD model of the $28 \%$ chordwise morphing trailing edge for high speed condition under skin structural constraint.

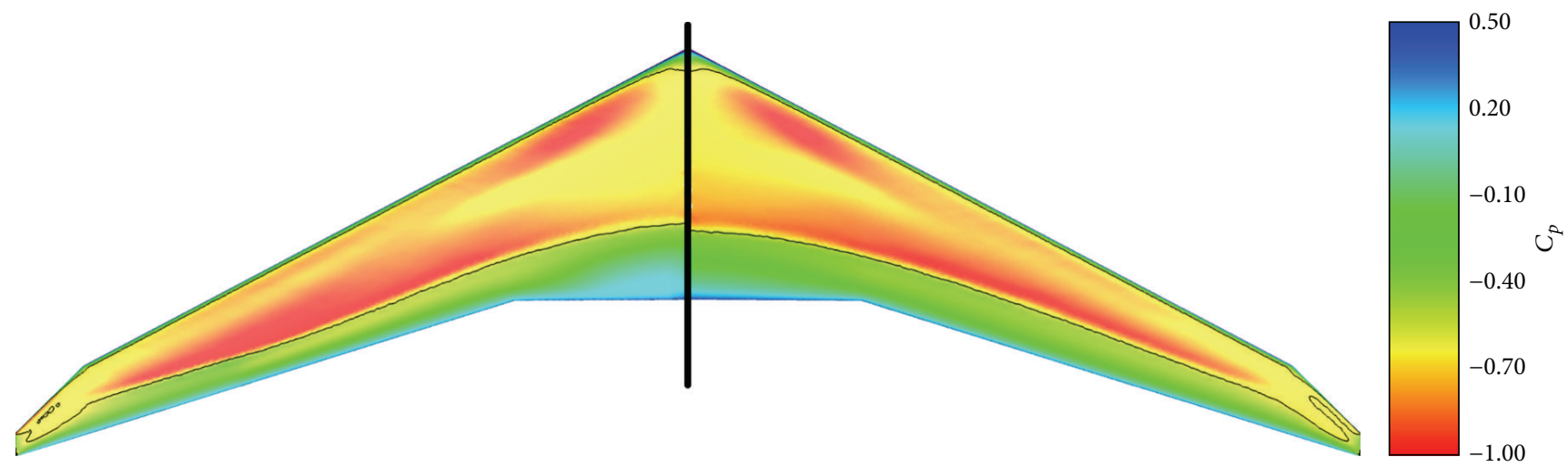

Figure 18: Comparison between the $C_{p}$ distributions at cruise Mach over the reference wing (right) and over the wing equipped with morphing trailing edge (left).

of morphing mechanisms based on compliant mechanisms. In particular the paper focused on the first level of the proposed multilevel design procedure. Indeed, the so-called knowledge-based shape optimization procedure has been introduced and described that is able to combine aerodynamic with structural performances, mainly related to the behavior of the skin during the shape variation. The proposed procedure has been evaluated in the design of morphing wing based on conformable leading and trailing edge surfaces able to adapt the wing camber during the mission profile. An external optimization loop works on the most important design variables that affect the camber morphing and it is dedicated to the aerodynamic performance evaluation. A nested loop, based on a particular development of CST method, guarantees that the outer loop works only on feasible shapes able to satisfy wing-box volume constraints and morphing skin requirements.

Some examples are reported, concerning the so-called Reference Aircraft, a regional-type aircraft developed inside NOVEMOR project and used as a test bench for a final assessment on morphing applications. The results obtained for the leading edge device at the subsonic regime and the trailing edge device at the transonic flight regime have been selected and the mission analyses have been completed to quantify possible benefits in terms of fuel saving. During these studies, only the aerodynamic behaviour and the structural behaviour of the morphing skin were considered. Neither the impact on the maximum takeoff weight of the morphing mechanisms nor the effect of aeroelasticity on the behavior of morphing devices has been considered. A family of optimal shapes have been produced and verified using both medium and high-fidelity tools showing promising results as well proving the versatility of the proposed approach. During the second level of the same procedure, all the optimal shapes can be adopted as multiple targets for the optimal design of the morphing mechanism, according to the multiobjective design strategy.

\section{Conflict of Interests}

The authors declare that there is no conflict of interests regarding the publication of this paper.

\section{Acknowledgments}

Special thanks go to Alexandre Antunes, Felipe Odaguil, and Grace Lima from EMBRAER for the CFD and mission analysis performed on Reference Aircraft. The research leading to these results has been partially funded by the European Union's Seventh Framework Programme [FP7/2007-2013] under Grant Agreement no. 285395. 


\section{References}

[1] L. Bréguet, "Aerodynamic efficiency and the reduction of air transport costs," Aeronautical Journal, vol. 26, pp. 307-313, 1922.

[2] M. Cavcar, "Bréguet range equation?" Journal of Aircraft, vol. 43, no. 5, pp. 1542-1544, 2006.

[3] A. De Gaspari, S. Ricci, L. Riccobene, and A. Scotti, "Active aeroelastic control over a multisurface wing: modeling and wind-tunnel testing," AIAA Journal, vol. 47, no. 9, pp. 1995-2010, 2009.

[4] H. P. Monner, M. Kintscher, T. Lorkowski, and S. Storm, "Design of a smart droop nose as leading edge high lift system for transportation aircrafts," in Proceedings of the 50th AIAA/ASME/ASCE/AHS/ASC Structures, Structural Dynamics and Materials Conference (SDM '09), AIAA 2009-2128, Palm Springs, Calif, USA, May 2009.

[5] J. J. Spillman, "The use of variable camber to reduce drag, weight and costs of transport aircraft," Aeronautical Journal, vol. 96, no. 951, pp. 1-9, 1992.

[6] A. McGowan, "Overview: morphing activities in the USA," in Proceedings of the Advanced Course on Morphing Aircraft Materials, Mechanisms and Systems, Lisbon, Portugal, November 2008.

[7] J. N. Kudva, "Overview of the DARPA smart wing project," Journal of Intelligent Material Systems and Structures, vol. 15, no. 4, pp. 261-267, 2004.

[8] D. Wagg, I. Bond, P. Weaver, and M. Friswell, Adaptive Structures: Engineering Applications, John Wiley \& Sons, 2007.

[9] C. Thill, J. Etches, I. Bond, K. Potter, and P. Weaver, "Morphing skins," The Aeronautical Journal, vol. 38, no. 10, 2008.

[10] D. Steenhuizen and M. van Tooren, "The implementation of a knowledge-based framework for the aerodynamic optimization of a morphing wing device," Advanced Engineering Informatics, vol. 26, no. 2, pp. 207-218, 2012.

[11] G. Molinari, A. F. Arrieta, and P. Ermanni, "Aero-structural optimization of 3-D adaptive wings with embedded smart actuators," in Proceedings of the 54th AIAA/ASME/ASCE/AHS/ASC Structures, Structural Dynamics and Materials Conference, Boston, Mass, USA, April 2013.

[12] P. D. Ciampa, T. Zill, T. Pfeiffer, and B. Nagel, "A functional shape parametrization approach for preliminary optimization of unconventional aircraft," in Proceedings of the 3rd CEAS Air \& Space Conference-21st AIDAA Congress, pp. 1513-1524, Venice, Italy, 2011.

[13] S. E. Gano, V. M. Pérez, J. E. Renaud, and S. M. Batill, "Multilevel variable fidelity optimization of a morphing unmanned aerial vehicle," in Proceedings of the 45th AIAA/ASME/ASCE/AHS/ASC Structures, Structural Dynamics and Materials Conference (AIAA '04), Palm Springs, Calif, USA, April 2004.

[14] S. Barbarino, O. Bilgen, R. M. Ajaj, M. I. Friswell, and D. J. Inman, "A review of morphing aircraft," Journal of Intelligent Material Systems and Structures, vol. 22, no. 9, pp. 823-877, 2011.

[15] A. De Gaspari and S. Ricci, "A two-level approach for the optimal design of morphing wings based on compliant structures," Journal of Intelligent Material Systems and Structures, vol. 22, no. 10, pp. 1091-1111, 2011.

[16] C. O. Johnston, D. A. Neal, L. D. Wiggins, H. H. Robertshaw, W. H. Mason, and D. J. Inman, "A model to compare the flight control energy requirements of morphing and conventionally actuated wings," in Proceedings of the 11th AIAA/ASME/AHS Adaptive Structures Conference (AIAA '03), Norfolk, Va, USA, April 2003.
[17] B. C. Prock, T. A. Weisshaar, and W. A. Crossley, "Morphing airfoil shape change optimization with minimum actuator energy as an objective," in Proceedings of the 9th AIAA/ISSMO Symposium on Multidisciplinary Analysis and Optimization (AIAA '02), Atlanta, Ga, USA, September 2002.

[18] H. Namgoong, W. A. Crossley, and A. S. Lyrintzis, "Aerodynamic optimization of a morphing airfoil using energy as an objective," in Proceedings of the 44th AIAA Aerospace Sciences Meeting and Exhibit (AIAA '06), Reno, Nev, USA, January 2006.

[19] P. Gamboa, J. Vale, F. J. P. Lau, and A. Suleman, “Optimization of a morphing wing based on coupled aerodynamic and structural constraints," AIAA Journal, vol. 47, no. 9, pp. 2087-2104, 2009.

[20] A. De Gaspari, S. Ricci, and L. Travaglini, "Aeroelastic analysis of a regional aircraft with active camber morphing device," in Proceedings of the International Forum on Aeroelasticity and Structural Dynamics (IFASD '15), Saint Petersburg, Russia, JuneJuly 2015.

[21] A. De Gaspari, A two levels approach for the optimal design of morphing wings based on compliant structures [Ph.D. thesis], Dipartimento di Ingegneria Aerospaziale, Politecnico di Milano, Milano, Italy, 2010.

[22] A. De Gaspari and S. Ricci, "Application of the active camber morphing concept based on compliant structures to a regional aircraft," in Industrial and Commercial Applications of Smart Structures Technologies, vol. 9059 of Proceedings of SPIE, pp. 121, San Diego, Calif, USA, April 2014.

[23] Y. He and R. K. Agarwal, "Shape optimization of NREL S809 airfoil for wind turbine blades using a multiobjective genetic algorithm," International Journal of Aerospace Engineering, vol. 2014, Article ID 864210, 13 pages, 2014.

[24] A. Shahrokhi and A. Jahangirian, "Airfoil shape parameterization for optimum Navier-Stokes design with genetic algorithm," Aerospace Science and Technology, vol. 11, no. 6, pp. 443-450, 2007.

[25] T. A. Weisshaar, "Morphing aircraft technology—new shapes for aircraft design," in Proceedings of the RTO-MP-AVT-141Multifunctional Structures/Integration of Sensors and Antennas, pp. 1-20, Neuilly-sur-Seine, France, October 2006.

[26] M. Secanell, A. Suleman, and P. Gamboa, "Design of a morphing airfoil using aerodynamic shape optimization," AIAA Journal, vol. 44, no. 7, pp. 1550-1562, 2006.

[27] B. M. Kulfan, "Universal parametric geometry representation method," Journal of Aircraft, vol. 45, no. 1, pp. 142-158, 2008.

[28] K. Deb, "Single and multi-objective optimization using evolutionary computation," in Proceedings of the 6th International Conference on Hydro-Informatics, pp. 14-35, Singapore, 2004.

[29] D. Goldberg, Genetic Algorithms in Search, Optimization and Machine Learning, Addison-Wesley, Boston, Mass, USA, 1989.

[30] A. Marco and J.-J. Martínez, "Polynomial least squares fitting in the Bernstein basis," Linear Algebra and its Applications, vol. 433, no. 7, pp. 1254-1264, 2010.

[31] Icem 12.1 Programmers Guide, ANSYS, 2008.

[32] FOI, CFD Flow Solver for Unstructured Grids, Division of Defence and Security, Systems and Technology, Department of Computational Physics, 2009, http://www.foi.se/edge.

[33] S. Lebofsky, E. Ting, and N. Nguyen, "Multidisciplinary drag optimization of reduced stiffness flexible wing aircraft with variable camber continuous trailing edge flap," in Proceedings of the 56th AIAA/ASCE/AHS/ASC Structures, Structural Dynamics, and Materials Conference, pp. 1-27, AIAA SciTech, Kissimmee, Fla, USA, January 2015. 
[34] S. Ghasemi, A. Mosahebi, and E. Laurendeau, "A twodimensional/infinite swept wing navier-stokes solver," in Proceedings of the 52nd Aerospace Sciences Meeting, pp. 1-11, AIAA SciTech, National Harbor, Md, USA, January 2014.

[35] M. Tomac and D. Eller, "From geometry to CFD gridsan automated approach for conceptual design," Progress in Aerospace Sciences, vol. 47, no. 8, pp. 589-596, 2011.

[36] M. Tomac and D. Eller, "Steps towards automated robust RANS meshing," in Proceedings of the 4th CEAS Air \& Space Conference, pp. 1-10, Linköping, Sweden, 2013.

[37] B. M. Kulfan, "Fundamental' parametric geometry representations for aircraft component shapes," in Proceedings of the 11th AIAA/ISSMO Multidisciplinary Analysis and Optimization Conference, Portsmouth, Va, USA, September 2006.

[38] F. Zhu and N. Qin, "Intuitive class/shape function parameterization for airfoils," AIAA Journal, vol. 52, no. 1, pp. 17-25, 2014.

[39] M. H. Straathof and M. J. L. van Tooren, "Extension to the class-shape-transformation method based on B-splines," AIAA Journal, vol. 49, no. 4, pp. 780-790, 2011.

[40] A. De Gaspari, S. Ricci, A. Antunes, F. Odaguil, and G. Lima, "Application of active camber morphing concept to a regional aircraft," in Proceedings of the 22nd AIAA/ASME/AHS Adaptive Structures Conference, AIAA 2014-125, pp. 1-23, National Harbor, Md, USA, January 2014. 

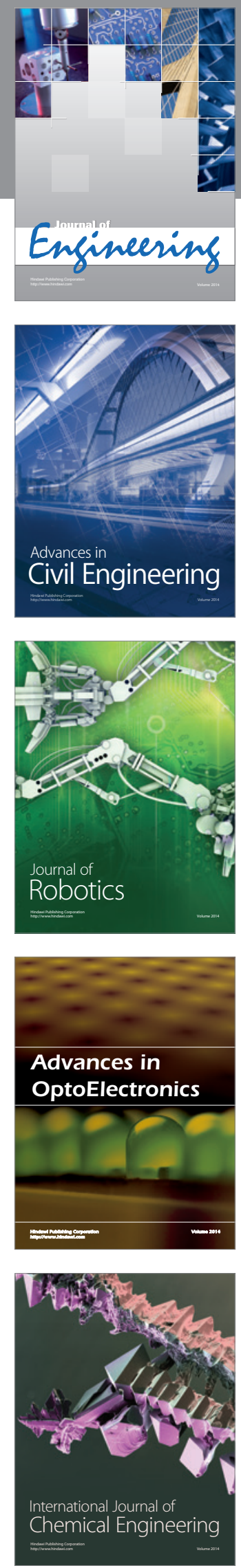

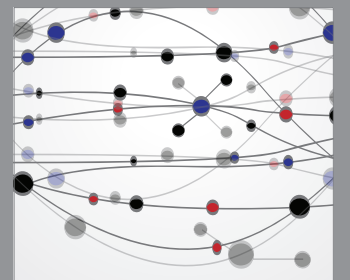

The Scientific World Journal
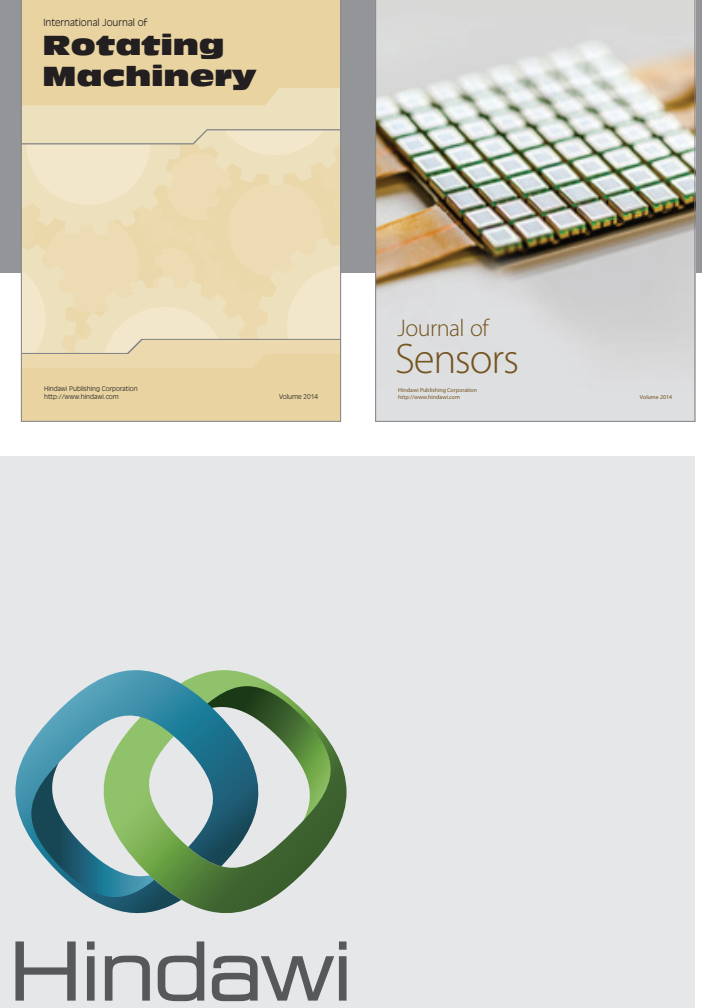

Submit your manuscripts at http://www.hindawi.com
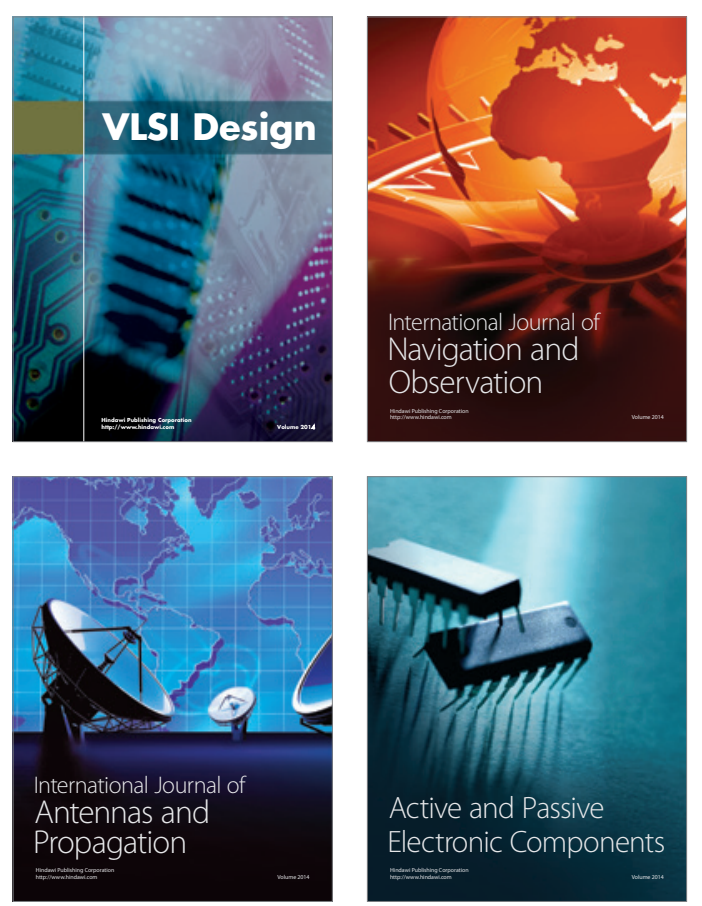
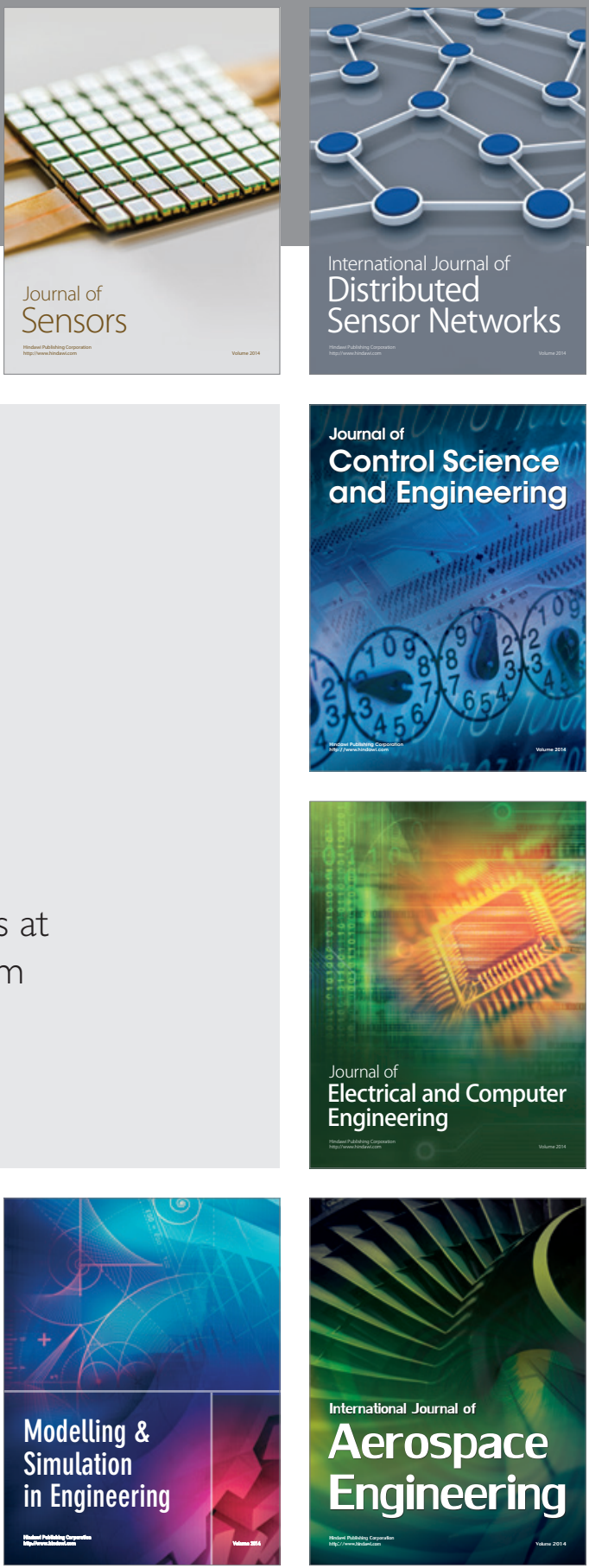

Journal of

Control Science

and Engineering
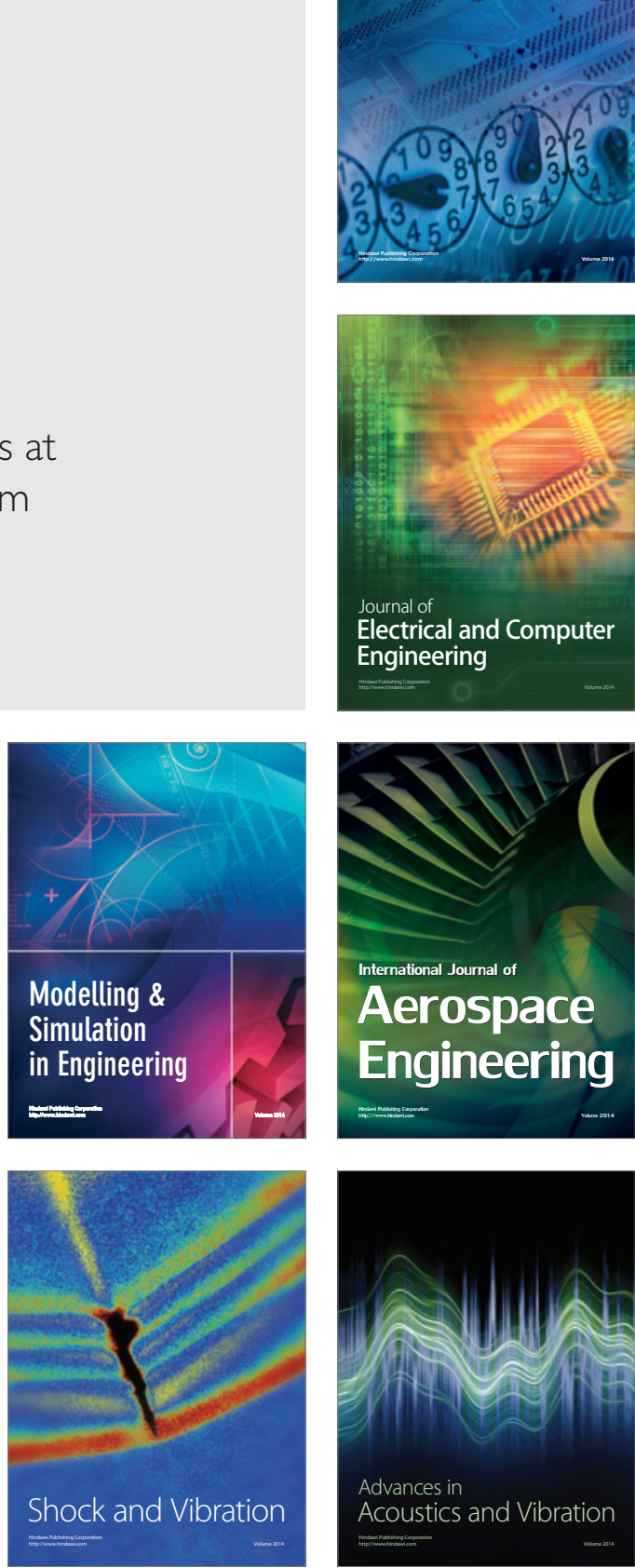